\title{
Conserved spin and orbital angular momentum Hall current in a two-dimensional electron system with Rashba and Dresselhaus spin-orbit coupling
}

\author{
Tsung-Wei Chen, ${ }^{1}$ Chih-Meng Huang, ${ }^{1}$ and G. Y. Guo ${ }^{1,2, *}$ \\ ${ }^{1}$ Department of Physics, National Taiwan University, Taipei 106, Taiwan \\ ${ }^{2}$ Department of Physics, Chinese University of Hong Kong, Shatin, N. T., Hong Kong \\ (Received 23 September 2005; revised manuscript received 29 March 2006; published 6 June 2006)
}

\begin{abstract}
We study theoretically the spin and orbital angular momentum (OAM) Hall effect in a high mobility two-dimensional electron system with Rashba and Dresselhuas spin-orbit coupling by introducing both the spin and OAM torque corrections, respectively, to the spin and OAM currents. We find that when both bands are occupied, the spin Hall conductivity is still a constant (i.e., independent of the carrier density) which, however, has an opposite sign to the previous value. The spin Hall conductivity in general would not be cancelled by the OAM Hall conductivity. The OAM Hall conductivity is also independent of the carrier density but depends on the strength ratio of the Rashba to Dresselhaus spin-orbit coupling, suggesting that one can manipulate the total Hall current through tuning the Rashba coupling by a gate voltage. We note that in a pure Rashba system, though the spin Hall conductivity is exactly cancelled by the OAM Hall conductivity due to the angular momentum conservation, the spin Hall effect could still manifest itself as nonzero magnetization Hall current and finite magnetization at the sample edges because the magnetic dipole moment associated with the spin of an electron is twice as large as that of the OAM. We also evaluate the electric field-induced OAM and discuss the origin of the OAM Hall current. Finally, we find that the spin and OAM Hall conductivities are closely related to the Berry vector (or gauge) potential.
\end{abstract}

DOI: 10.1103/PhysRevB.73.235309

PACS number(s): 73.43.Cd, 73.63.Hs, 75.47.- m, 85.75.-d

\section{INTRODUCTION}

Spin transport electronics or spintronics in semiconductors has become a very active research field in condensed matter mainly because of its potential applications in information storage and processing and other electronic technologies ${ }^{1}$ and also because of many fundamental questions on the physics of electron spin. ${ }^{2}$ Spin current generation is an important issue in the emerging spintronics. Recent proposals of the intrinsic spin Hall effect are therefore remarkable. ${ }^{3,4}$ In the spin Hall effect, a transverse spin current is generated in response to an electric field in a metal with relativistic electron interaction (spin-orbit coupling). This effect has been considered to arise extrinsically, i.e., by impurity scattering. ${ }^{5}$ The scattering becomes spin dependent in the presence of spin-orbit coupling, and this gives rise to the spin Hall effect. In the recent proposals, in contrast, the spin Hall effect can arise intrinsically in hole-doped ( $p$-type) bulk semiconductors ${ }^{3}$ and also in electron-doped (n-type) semiconductor heterostructures ${ }^{4}$ due to intrinsic spin-orbit coupling in the band structure. This intrinsic spin Hall effect offers an exciting possibility of pure electric driven spintronics in semiconductors, where spin-orbit coupling is relatively strong and which can be more readily integrated with welldeveloped semiconductor electronics.

A large number of theoretical papers have been written addressing various issues about the intrinsic spin Hall effect. In Ref. 6, a systematic semiclassical theory of spin transport is presented, resolving a discrepancy between the prediction of Ref. 3 and the Kubo formula result. In Ref. 7, an orbitalangular-momentum (OAM) Hall current is predicted to exist in response to an electric field and is found to cancel exactly the spin Hall current in the spin Hall effect. In Ref. 8, however, $a b$ inito relativistic band structure calculations show that the OAM Hall conductivity in hole-doped semiconductors is one order of magnitude smaller than the spin Hall conductivity, indicating no cancellation between the spin and OAM Hall effects in bulk semiconductors because of the orbital quenching by the cubic crystalline anisotropy. There is also an intensive debate about whether the intrinsic spin Hall effect remains valid beyond the ballistic transport regime. ${ }^{9-11}$ On the other hand, experimental measurements of large spin Hall effects for the Rashba two-dimensional electron gas and for $n$-type bulk semiconductors have just been reported, ${ }^{12,13}$ although more work is needed to firmly establish the intrinsic or extrinsic nature of the results.

At present, an urgent current issue in spintronics research is about the appropriate definition of the spin current. ${ }^{14-18}$ In almost all previous studies of the intrinsic spin Hall effect, the spin current is intuitively defined as the expectation value of the spin and velocity operators, namely, $\frac{1}{2}\left\{\mathbf{v}, s_{z}\right\}$, where $\{$, is the anticommutator defined by $\{A, B\}=A B+B A$. Similarly, the OAM current is defined as the expectation value of $\frac{1}{2}\left\{\mathbf{v}, L_{z}\right\}$. Here, $s_{z}$ and $L_{z}$ are the $z$ component of spin and OAM operators, respectively, and $\mathbf{v}$ is the velocity operator. However, this conventionally defined spin (OAM) current is not conserved in systems with spin-orbit interaction. ${ }^{6}$ Consequently, many fundamental questions on the intrinsic spin Hall effect in semiconductors remain unresolved. Very recently, in Ref. 16, a proper definition of the conserved effective spin current is established for systems with spin-orbit coupling, and the conserved spin current density is defined as

$$
\mathcal{J}_{s}=\mathbf{J}_{s}+\mathbf{P}_{\tau},
$$

where $\mathbf{J}_{s}=\operatorname{Re}\left[\Psi^{\dagger} \frac{1}{2}\left\{\mathbf{v}, s_{z}\right\} \Psi\right]$ is conventional spin current density and $\mathbf{P}_{\tau}$ is the torque dipole density which arise from spin torque $\mathcal{T}_{z}^{s}=\operatorname{Re}\left[\Psi^{\dagger} \frac{1}{i \hbar}\left[s_{z}, H_{0}\right] \Psi\right]$ where $H_{0}$ is the Hamiltonian 
of the system. The commutator [,] is defined by $[A, B]$ $=A B-B A$. The effective spin current density $\mathcal{J}_{s}$ then satisfies the standard continuity equation ${ }^{16}$ of $\frac{\partial \mathcal{S}_{z}}{\partial t}+\nabla \cdot \mathcal{J}_{s}=0$ where the spin density is defined by $\mathcal{S}_{z}=\Psi^{\dagger} s_{z} \Psi$. A derivation of the effective spin continuity equation in Rashba-Dresselhaus system is given in Appendix A. Within this definition of the effective spin current, the unphysical intrinsic spin Hall effect in insulators with localized orbitals vanishes, and the Onsager relation between the spin Hall effect and inverse spin Hall effect is ensured. ${ }^{16}$ Furthermore, this definition of the spin current predicts opposite signs of spin Hall coefficients for a couple of semiconductor models such as the Rashba and $k$-cubed Rashba Hamiltonians. ${ }^{16}$

In this paper, we extend the theory proposed in Ref. 16 to two-dimensional electron systems with both Rashba and Dresselhaus spin-orbit coupling. Furthermore, we introduce the concept of the effective OAM current by including an OAM torque correction term, and investigate the OAM Hall effect in both pure Rashba system and systems with RashbaDresselhaus spin-orbit coupling. We derive the effective OAM continuity equation for the Rashba-Dresselhaus system (Appendix B). Also in this paper, we argue that in a pure Rashba system, though the OAM Hall conductivity is found to exactly cancel the spin Hall conductivity, there nevertheless would be nonzero magnetization current and finite magnetization at the sample edges because the magnetic dipole moment associated with spin angular moment is twice as large as that of the OAM. Finally, we find that there are interesting relations between the Berry vector potential and the spin and OAM Hall conductivities.

\section{MODEL HAMILTONIAN AND LINEAR RESPONSE CALCULATION}

\section{A. Rashba-Dresselhaus Hamiltonian}

For a two-dimensional electron gas (2DEG) confined in a semiconductor heterostructure, two major spin-orbit (SO) interaction terms are usually present. One is the Rashba term, ${ }^{19}$

$$
H_{R}=\frac{\lambda}{\hbar} \vec{\sigma} \cdot\left(\mathbf{p} \times \hat{e}_{z}\right),
$$

which stems from the structural inversion asymmetry. Here, $\vec{\sigma}=\left(\sigma_{x}, \sigma_{y}\right)$ and $\sigma_{z}$ are the three Pauli matrices. The other is the Dresselhaus SO coupling which results from the bulk inversion asymmetry, if the heterostructure is made of semiconductors without spatial inversion symmetry such as semiconductors in the zincblende structure. ${ }^{20}$ The Dresselhaus term is given by

$$
H_{D}=-\frac{\beta}{\hbar}\left(p_{x} \sigma_{x}-p_{y} \sigma_{y}\right) .
$$

Therefore, the full Hamiltonian for the 2DEG with RashbaDresselhaus SO coupling can be written as

$$
H_{0}=\frac{p^{2}}{2 m}+H_{R}+H_{D}
$$

where $m$ is the effective mass of the electrons in the 2DEG. Interestingly, though the Dresselhaus coupling coefficient $\beta$ is fixed for a given structure, the Rashba coupling strength $\lambda$ can be tuned by a gate voltage by up to $50 \%,{ }^{21}$ thereby providing an opportunity to study the interesting interplay between both types of the SO coupling. The Hamiltonian has been solved exactly by several authors. The eigenstates can be written as

$$
|n \mathbf{k}\rangle=\frac{1}{\sqrt{2}}\left(\begin{array}{c}
e^{-i \theta(\mathbf{k})} \\
\text { in }
\end{array}\right),
$$

where $n= \pm 1$ is band index and

$$
\tan \theta(\mathbf{k})=\frac{\lambda k_{y}-\beta k_{x}}{\lambda k_{x}-\beta k_{y}} .
$$

The corresponding eigenenergies is given by

$$
E_{n \mathbf{k}}=\frac{\hbar^{2}}{2 m} k^{2}-n k \gamma(\phi),
$$

where $\mathbf{k}=\left(k_{x}, k_{y}\right), k=|\mathbf{k}|, \tan (\phi)=k_{y} / k_{x}, \cos \theta(\mathbf{k})=(\lambda \cos \phi$ $-\beta \sin \phi) / \gamma(\phi), \quad \sin \theta(\mathbf{k})=(\lambda \sin \phi-\beta \cos \phi) / \gamma(\phi), \quad$ and $\gamma(\phi)=\sqrt{\left(\lambda^{2}+\beta^{2}\right)-2 \lambda \beta \sin (2 \phi)}$. It can be proved that the difference of two Fermi momenta $\hbar k_{F}^{ \pm}$is given by $\left(k_{F}^{+}-k_{F}^{-}\right)$ $=2 m \gamma(\phi) / \hbar^{2}$. The band structure consists of two energy bands which are degenerate at the center of the 2D momentum space $\mathbf{k}=0$, as schematically illustrated in the left-hand panel of Fig. 1(a). The spins associated with the eigenstates all lie in the $x y$ plane, as shown in the right-hand panel of Fig. 1(a) and in the left-hand panel of Fig. 1(b).

\section{B. Spin Hall conductivity}

Let us consider a uniform electric field $\mathbf{E}$ applied in the $y$ direction. The total Hamiltonian in this case is given by $H$ $=H_{0}+e E y$, where $-e$ is the electron charge. Let us treat the term $e E y$ as a small perturbation. To determine the spin transport coefficient, we start with the linear response Kubo formula in the clean limit. The conventional spin Hall conductivity is ${ }^{22}$

$$
\begin{aligned}
\sigma_{x y}^{s_{0}}= & -\frac{e \hbar}{V} \sum_{n \neq n^{\prime}} \sum_{\mathbf{k}}\left(f_{n \mathbf{k}}-f_{n^{\prime} \mathbf{k}}\right) \\
& \times \frac{\operatorname{Im}\left[\left\langle n \mathbf{k}\left|j_{x}^{s_{z}}\right| n^{\prime} \mathbf{k}\right\rangle\left\langle n^{\prime} \mathbf{k}\left|v_{y}\right| n \mathbf{k}\right\rangle\right]}{\left(E_{n \mathbf{k}}-E_{n^{\prime} \mathbf{k}}\right)^{2}},
\end{aligned}
$$

where $j_{x}^{s_{z}}=1 / 2\left\{v_{x}, s_{z}\right\}$ is the usual spin current operator, $\mathbf{v}(\mathbf{k})=\partial H_{0}(\mathbf{k}) / \hbar \partial \mathbf{k}, H_{0}(\mathbf{k})=e^{-i \mathbf{k} \cdot \mathbf{x}} H_{0} e^{i \mathbf{k} \cdot \mathbf{x}}, s_{z}=\frac{\hbar}{2} \sigma_{z},|n \mathbf{k}\rangle$ is the eigenstate of the $n$th band with momentum $\mathbf{k}$, and $f_{n \mathbf{k}}$ is the Fermi-Dirac distribution. The index $n \neq n^{\prime}$ denotes no intraband transition. Similarly, the spin torque response coefficient can also be obtained, ${ }^{16}$

$$
\begin{aligned}
\chi(\mathbf{q})= & \frac{i e \hbar}{V} \sum_{n \neq n^{\prime}} \sum_{\mathbf{k}}\left(f_{n \mathbf{k}}-f_{n^{\prime} \mathbf{k}+\mathbf{q}}\right) \\
& \times \frac{\left\langle n \mathbf{k}\left|\tau_{s}(\mathbf{k}, \mathbf{q})\right| n^{\prime} \mathbf{k}+\mathbf{q}\right\rangle\left\langle n^{\prime} \mathbf{k}+\mathbf{q}|\mathbf{v}(\mathbf{k}, \mathbf{q})| n \mathbf{k}\right\rangle}{\left(E_{n \mathbf{k}}-E_{n^{\prime} \mathbf{k}+\mathbf{q}}\right)^{2}},
\end{aligned}
$$

where $\quad \tau_{s}(\mathbf{k}, \mathbf{q})=\frac{1}{2}\left[\tau_{s}(\mathbf{k})+\tau_{s}(\mathbf{k}+\mathbf{q})\right], \quad \mathbf{v}(\mathbf{k}, \mathbf{q})=\frac{1}{2}[\mathbf{v}(\mathbf{k})+\mathbf{v}(\mathbf{k}$ 


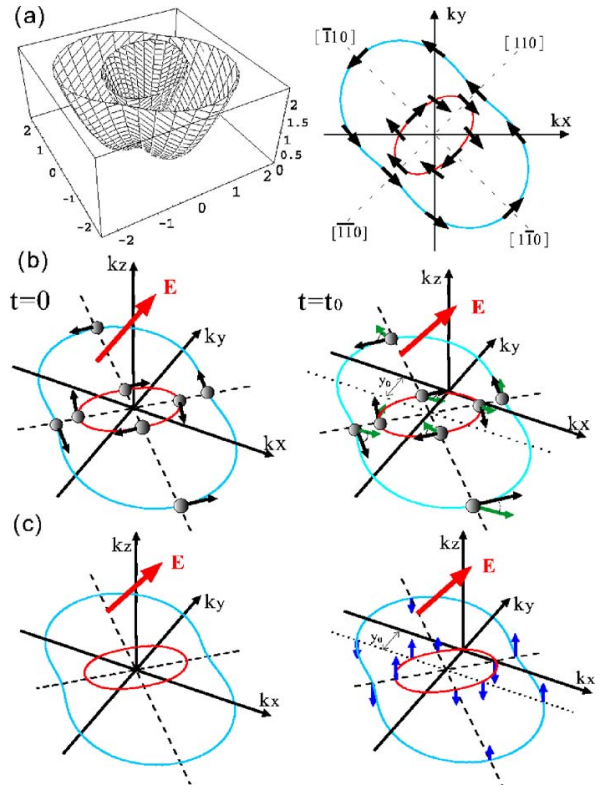

FIG. 1. (Color online) (a) The schematic band structure of the Rashba-Dresselhaus spin-orbit coupling system with $|\lambda| \neq|\beta|$. The outer sheet is for $n=+1$ and the inner sheet, for $n=-1$. The two sheets touch at $\mathbf{k}=0$. If $\lambda=\beta$, the sheets become degenerate at line $k_{x}=k_{y}$ (see, also, Fig. 1 in Ref. 23). The right-hand panel is the top view of the two bands. The arrows labeled on the two bands denote the spin directions of the associated $k$ points, respectively. (b) Initially, the spins are in the $x y$ plane (the left-hand panel). At a short time $t_{0}$ after an external electric field $E$ [indicated as the large horizontal (red) arrow] is applied along the $y$ axis, the bands move in the $-k_{y}$ direction with the distance $\delta k_{y}=e E t_{0} / \hbar$ (see Sec. II E). Each spinor feels an effective magnetic field and will do precession. For $k_{x}>0$, the spins tend to tilt down and for $k_{x}<0$, the spins tend to tilt up (the right-hand panel). The $z$ component of the spin is given by $\delta\left\langle s_{z}\right\rangle=-e E \hbar\left(\lambda^{2}-\beta^{2}\right) \cos \phi / 4 n k^{2} \gamma^{3}(\phi)$ (c). Before an external electric field is applied, all the eigenstates carry zero orbital angular momentum (OAM), i.e., $\left\langle L_{z}\right\rangle_{0}=0$ (see Sec. II E). When the electric field is turned on, the $z$ component of the OAM is induced as shown in the right-hand panel (small vertical arrows). A linear response calculation would give the $z$ component as $\delta\left\langle L_{z}\right\rangle=e E \hbar\left(\lambda^{2}\right.$ $\left.-\beta^{2}\right)^{2} \cos \phi / 4 n k^{2} \gamma^{5}(\phi)$.

$+\mathbf{q})]$ with $\tau_{s}=\frac{1}{i \hbar}\left[s_{z}, H_{0}\right]$. In the above equation, $\sigma_{x y}^{s_{0}}$ is the transport coefficient of conventional spin current and $\sigma_{x y}^{\tau_{s}}$ can be determined from the dc response of the spin torque dipole: $\quad \mathbf{P}_{\tau}=\left.\operatorname{Re}\left[i \nabla_{\mathbf{q}} \chi(\mathbf{q})\right]\right|_{\mathbf{q} \rightarrow 0} \cdot \mathbf{E}, \quad$ namely, $\quad \sigma_{x y}^{\tau_{s}}$ $=\left.\operatorname{Re}\left[i \partial \chi_{y}(\mathbf{q}) / \partial q_{x}\right]\right|_{q_{x} \rightarrow 0}{ }^{16}$ Here $\chi(\mathbf{q})$ is the response coefficient of spin torque to an electric field with finite wave vector q. Before doing summation in the above Kubo formula, we expand the term inside the summation to the first order of $q$. At zero temperature, $f_{n^{\prime} \mathbf{k}+\mathbf{q}} \simeq f_{n^{\prime} \mathbf{k}}$ $+\mathbf{q} \cdot\left(\partial E_{n^{\prime} \mathbf{k}} / \partial \mathbf{k}\right) \partial f_{n^{\prime} \mathbf{k}} / \partial E_{n^{\prime} \mathbf{k}}$, where the first term is a step function while the second term was involved with a Dirac delta function. We assume that the two bands are all occupied by the electrons and the Fermi energy is larger than the SO splitting. The spin Hall conductivity is

$$
\sigma_{x y}^{s}=\sigma_{x y}^{s_{0}}+\sigma_{x y}^{\tau_{s}},
$$

which is the linear response of the conserved effective spin current $\mathcal{J}_{s}$ to the electric field. ${ }^{16}$ It is straightforward to cal- culate the spin Hall conductivity (the detailed derivation is given in Appendix $\mathrm{C})$. We find that $\sigma_{x y}^{s_{0}}=-\operatorname{sign}\left(\lambda^{2}\right.$ $\left.-\beta^{2}\right) e / 8 \pi$ is the conventional spin Hall conductivity and $\sigma_{x y}^{\tau_{s}}=\operatorname{sign}\left(\lambda^{2}-\beta^{2}\right) e / 4 \pi$ is the conductivity due to the spin torque correction. Therefore, the spin Hall conductivity defined as the response of the conserved effective spin current is

$$
\sigma_{x y}^{s}=\left\{\begin{array}{cc}
\frac{e}{8 \pi}, & \lambda^{2}>\beta^{2}, \\
0, & \lambda^{2}=\beta^{2}, \\
-\frac{e}{8 \pi}, & \lambda^{2}<\beta^{2},
\end{array}\right.
$$

which is independent on the carrier density. Remarkably, with the spin torque correction term $\sigma_{x y}^{\tau_{s}}$ included, the sign of the effective spin Hall conductivity is opposite to the conventional spin Hall conductivity reported in Refs. 23 and 24.

\section{Orbital angular momentum Hall conductivity}

The usual OAM current operator was introduced as

$$
j_{x}^{o}=\frac{1}{2}\left\{v_{x}, L_{z}\right\}
$$

where $L_{z}$ is the $z$ component of the OAM operator. In the Rashba Hamiltonian and with this definition of the OAM current, the spin Hall effect is always accompanied by the OAM Hall effect which was first noted by Zhang and Yang. ${ }^{7}$ In a SO system, such a definition has the same problems as the conventional spin current operator because the OAM current is not a conserved quantity. Therefore, we derive the continuity equation for the effective OAM current (see Appendix B). We find $\frac{\partial \mathcal{L}_{z}}{\partial t}+\nabla \cdot \mathbf{J}_{o}=\mathcal{T}_{z}^{o}$, where $\mathcal{L}_{z}=\Psi^{\dagger} L_{z} \Psi$ is the $z$ component of the OAM density, $\mathbf{J}_{o}=\operatorname{Re}\left[\Psi^{\dagger} \mathbf{j}^{o_{0}} \Psi\right]$ is the OAM current density and $\mathbf{j}^{o_{0}}=\frac{1}{2}\left\{\mathbf{v}, L_{z}\right\} . \mathcal{T}_{z}^{o}=\operatorname{Re}\left[\Psi^{\dagger} \tau_{o} \Psi\right]$ is the torque density, where $\tau_{o}=\frac{1}{i \hbar}\left[L_{z}, H_{0}\right]$. In analogue to the conserved effective spin current operator proposed in Ref. 16 , the average torque in the bulk is zero, and hence we have $\frac{1}{V} \int d V \mathcal{T}_{z}^{o}=0$. The torque density can be written as the divergence of torque dipole density $\mathbf{P}_{\tau}^{o}(\mathbf{x})$, i.e., $\mathcal{T}_{z}^{o}=-\nabla \cdot \mathbf{P}_{\tau}^{o}(\mathbf{x})$. Substituting $\mathcal{T}_{z}^{o}=-\nabla \cdot \mathbf{P}_{\tau}^{o}$ into continuity equation of OAM, we obtain $\mathcal{J}_{o}=\mathbf{J}_{o}+\mathbf{P}_{\tau}^{o}$ as the effective OAM current. The torque dipole density vanishes outside the bulk, and we can write $\int_{V} d V \mathbf{P}_{\tau}^{o}=\int_{V} d V\left(-\mathbf{x} \nabla \cdot \mathbf{P}_{\tau}^{o}\right)=\int_{V} d V \mathbf{x} \mathcal{T}_{z}^{o}$. Therefore, the unique form of torque dipole density is $\ddot{\mathbf{P}}_{\tau}^{o}=\operatorname{Re}\left[\Psi^{\dagger}\left(\mathbf{x} \tau_{o}\right) \Psi\right]$. In short, we may define an effective OAM current operator,

$$
\mathcal{J}_{o}=\frac{d \mathbf{x}}{d t} L_{z}+\mathbf{x} \tau_{o}
$$

It has an extra term $\mathbf{x} \tau_{o}$ which is the correction term due to the OAM torque. The corresponding OAM current density $\mathcal{J}_{o}=\operatorname{Re} \Psi^{\dagger}(\mathbf{x}) \hat{\mathcal{J}}_{o} \Psi(\mathbf{x})$ defined as the expectation value of this current operator satisfies the standard continuity equation of $\frac{\partial \mathcal{L}_{z}}{\partial t}+\nabla \cdot \mathcal{J}_{o}=0$. As for the spin Hall conductivity, ${ }^{16}$ the OAM Hall conductivity has two parts, 


$$
\sigma_{x y}^{o}=\sigma_{x y}^{o}+\sigma_{x y}^{\tau_{o}},
$$

where the first term is the usual OAM Hall conductivity and the second term comes from the OAM torque correction. Thus, the OAM Hall conductivity can be calculated from Eq. (8) and (9) by substituting $j_{x}^{s_{z}}$ and $\tau_{s}$ with $j_{x}^{o_{0}}$ and $\tau_{o}$ $=\frac{1}{i \hbar}\left[L_{z}, H_{0}\right]$, respectively. It can be shown (Appendix $\left.\mathrm{C}\right)$ that the OAM Hall conductivity defined as the linear response of the effective OAM current is

$$
\sigma_{x y}^{o}=\left\{\begin{array}{cc}
-\frac{\lambda^{2}+\beta^{2}}{\lambda^{2}-\beta^{2}} \frac{e}{8 \pi}, & \lambda^{2}>\beta^{2}, \\
0, & \lambda^{2}=\beta^{2}, \\
\frac{\lambda^{2}+\beta^{2}}{\lambda^{2}-\beta^{2}} \frac{e}{8 \pi}, & \lambda^{2}<\beta^{2}
\end{array}\right.
$$

which is a function of the ratio $\lambda / \beta$ and is also independent of the carrier density.

\section{Berry vector potential and Hall conductivity}

When the Hamiltonian of a physical system is parametrized by periodically changing environment, the state ket of system will travel on a close path and return to initial state ket after a period. The final state ket must coincide with initial state vector, apart from a phase factor. Berry ${ }^{25}$ has shown that the state ket will acquire an additional phase factor as the system undergoes the adiabatic evolution. The phase factor accompanying the adiabatic evolution is called Berry phase. Berry phase cannot be removed by any gauge transformation for a closed path. ${ }^{26}$ In solids, the Bloch state also acquire a Berry phase if the applied perturbation can make a constraint such that $\mathbf{k}$ travels adiabatically on a closed path in the Brillouin zone. ${ }^{27}$ The Berry phase of Bloch states $|n \mathbf{k}\rangle$ for a closed path $C$ can be expressed by

$$
\Phi_{n}=\oint_{c} \mathbf{A}_{n}(\mathbf{k}) \cdot d \mathbf{l},
$$

where $\mathbf{A}_{n}(\mathbf{k})=\left\langle n \mathbf{k}\left|(-i) \frac{\partial}{\partial \mathbf{k}}\right| n \mathbf{k}\right\rangle$ is defined as Berry vector potential (or Berry connection). Berry curvature is defined as $\Omega_{n}=\nabla_{\mathbf{k}} \times \mathbf{A}_{n}$. The Berry vector potential and curvature are the salient characteristic of energy band structure and hence have important applications in transport properties of carriers. It has been shown that the equation of motion of Bloch electron has an extra anomalous velocity in terms of Berry curvature of Bloch states. ${ }^{28}$ In bulk $p$-type semiconductors with spin-orbit coupling, the existence of $k$-space magnetic monopole (Berry curvature) in the degeneracy $\Gamma$ point of band structure results in a transverse force exerting on spin. ${ }^{3}$ The connection between the dissipationless spin Hall conductivity and Berry curvature has been shown in Ref. 29. Here we further show that in two-dimensional RashbaDresselhaus systems, we can write the spin Hall and OAM Hall conductivities in terms of Berry curvature and vector potential.

With the eigenstates $|n \mathbf{k}\rangle$ given by Eq. (5), we can prove that the matrix element $\left\langle n^{\prime} \mathbf{k}\left|v_{y}\right| n \mathbf{k}\right\rangle$ satisfies the following relation $\left(\right.$ for $n^{\prime} \neq n$ ):

$$
\left\langle n^{\prime} \mathbf{k}\left|v_{y}\right| n \mathbf{k}\right\rangle=\frac{i}{\hbar}\left(E_{n \mathbf{k}}-E_{n^{\prime} \mathbf{k}}\right)\left\langle n^{\prime} \mathbf{k}\left|(-i) \frac{\partial}{\partial k_{y}}\right| n \mathbf{k}\right\rangle,
$$

where $\left\langle n^{\prime} \mathbf{k}\left|(-i) \frac{\partial}{\partial k_{y}}\right| n \mathbf{k}\right\rangle=-\frac{1}{2} \frac{\partial \theta}{\partial k_{y}}$. The Berry vector potential is then given by

$$
\mathbf{A}=\left\langle n \mathbf{k}\left|(-i) \frac{\partial}{\partial \mathbf{k}}\right| n \mathbf{k}\right\rangle=-\frac{1}{2} \frac{\partial \theta}{\partial \mathbf{k}} .
$$

Substituting Eqs. (17) and (18), into Eq. (8) and noting that $\sigma_{x y}^{s_{0}}=-\sigma_{y x}^{s_{0}},{ }^{30}$ we have

$$
\sigma_{x y}^{s_{0}}=-\frac{e \hbar}{2 m V} \sum_{n} \sum_{\mathbf{k}} \frac{f_{n \mathbf{k}}}{\omega_{n \mathbf{k}}}[\mathbf{k} \times \mathbf{A}]_{z}
$$

where $\omega_{n \mathbf{k}}$ is defined as $\omega_{n \mathbf{k}} \equiv\left(E_{n \mathbf{k}}-E_{-n \mathbf{k}}\right) / \hbar$. Similarly, we also obtain,

$$
\sigma_{x y}^{o_{0}}=-\frac{e \hbar}{m V} \sum_{n} \sum_{\mathbf{k}} \frac{f_{n \mathbf{k}}}{\omega_{n \mathbf{k}}}[\mathbf{k} \times \mathbf{A}]_{z}^{2},
$$

where $\sigma_{x y}^{o_{0}}=-\sigma_{y x}^{o_{0}}$ was used. ${ }^{30}$ The spin Hall and OAM Hall conductivities are therefore related to Berry vector potential. The Berry curvature is $\Omega(\mathbf{k})_{z}=\left[\nabla_{\mathbf{k}} \times \mathbf{A}\right]_{z}=-\frac{\left(\lambda^{2}-\beta^{2}\right)}{\left|\lambda^{2}-\beta^{2}\right|} \pi \delta^{(2)}(\mathbf{k})$. The corresponding Berry phase is then given by the path integral of the Berry vector potential, i.e., $\Phi=\oint_{C} d \mathbf{l} \cdot \mathbf{A}$ $=\int_{R} \Omega(\mathbf{k})_{z} d k_{x} d k_{y}=-\frac{\lambda^{2}-\beta^{2}}{\left|\lambda^{2}-\beta^{2}\right|} \pi=-\operatorname{sign}\left(\lambda^{2}-\beta^{2}\right) \pi$, where $R$ is the region containing the origin in the $\mathbf{k}$ plane. The Berry curvature $\Omega_{z}$ is zero for $\mathbf{k} \neq 0$ but nonzero for $\mathbf{k}=0$ where the two bands of the Rashba-Dresselhaus system are degenerate. As for the spin transverse force for the Luttinger Hamiltonian given in Ref. 29, the corresponding spin transverse force in Rashba-Dresselhaus system can also be given in terms of the Berry curvature. Even though the Berry curvature vanishes for $\mathbf{k} \neq 0$, the spin Hall current can nonetheless occur due to the Aharonov-Bohm-type effect at $\mathbf{k}=0 .{ }^{31}$

Let us now discuss the effects of the different choice of the eigenstates on the above findings. In, e.g., Ref. 32, the eigenstates $(|n \tilde{\mathbf{k}}\rangle)$

$$
|+\tilde{\mathbf{k}}\rangle=\frac{1}{\sqrt{2}}\left(\begin{array}{c}
-i e^{-i \theta} \\
1
\end{array}\right), \quad|-\tilde{\mathbf{k}}\rangle=\frac{1}{\sqrt{2}}\left(\begin{array}{c}
1 \\
-i e^{i \theta}
\end{array}\right)
$$

were used for the Rashba-Dresselhaus system. We find that the unitary transformation defined by the matrix

$$
U=\frac{1}{2}\left(\begin{array}{cc}
-i+e^{i \theta} & i-e^{-i \theta} \\
e^{i \theta}-i e^{2 i \theta} & -i+e^{i \theta}
\end{array}\right)
$$

with $U U^{\dagger}=U^{\dagger} U=1$, will transform the eigenstates of Eq. (5) to that of Eq. (21), i.e., $|n \tilde{\mathbf{k}}\rangle=U|n \mathbf{k}\rangle$. Using the eigenstates in Eq. (21), the Berry vector potential is given by

$$
\mathbf{A}_{+} \equiv\left\langle+\tilde{\mathbf{k}}\left|(-i) \frac{\partial}{\partial \mathbf{k}}\right|+\tilde{\mathbf{k}}\right\rangle=-\frac{1}{2} \frac{\partial \theta}{\partial \mathbf{k}}
$$




$$
\mathbf{A}_{-} \equiv\left\langle-\tilde{\mathbf{k}}\left|(-i) \frac{\partial}{\partial \mathbf{k}}\right|-\tilde{\mathbf{k}}\right\rangle=+\frac{1}{2} \frac{\partial \theta}{\partial \mathbf{k}},
$$

that depends on the band index $n$. We may define $\mathbf{A}_{n}=n \mathbf{A}$ for both the bases of (5) and (21). As a result, the Berry phase given by Eq. (16) also depends on the band index $n,{ }^{32}$ i.e.,

$$
\Phi_{ \pm}=\oint_{c} \mathbf{A}_{ \pm}(\mathbf{k}) \cdot d \mathbf{l}=\mp \operatorname{sign}\left(\lambda^{2}-\beta^{2}\right) \pi= \pm \Phi,
$$

where $\Phi$ is the Berry phase when the basis of Eq. (5) is used. In contrast, the signs of the Berry phase $\Phi$ for the two bands are the same in the choice of the eigenstates of Eq. (5). Nevertheless, we can write $\Phi_{n}=n \Phi$ with $n= \pm 1$. Note that the charge Hall effect would be zero when the basis of Eq. (21) is used, and would be nonzero if the basis of Eq. (5) is chosen, as have been pointed out in Ref. 32.

Using $\mathbf{A}=-\frac{1}{2} \frac{\partial \theta}{\partial \mathbf{k}}$ [Eq. (18)], we can show that $\mathbf{A}=[\mathbf{k}$ $\times \mathbf{A}]_{z} \mathbf{a}_{0}$, where $\mathbf{a}_{0} \equiv\left(-\frac{k_{y}}{k^{2}}, \frac{k_{x}}{k^{2}}\right)=\frac{\hat{e}_{\phi}}{k} \quad$ and $\quad[\mathbf{k} \times \mathbf{A}]_{z}=-\left(\lambda^{2}\right.$ $\left.-\beta^{2}\right) / 2 \gamma^{2}(\phi)$ which depends only on $\phi$. After some vector algebra calculations, the spin Hall conductivity in Eq. (19) can be written as

$$
\sigma_{x y}^{s_{0}}=\frac{e}{8 \pi^{2}} \sum_{n} \oint_{C} d \mathbf{l} \cdot n[\mathbf{k} \times \mathbf{A}]_{z} \mathbf{a}_{0} \frac{k_{F}^{n}}{k_{F}^{+}-k_{F}^{-}},
$$

where $\left(k_{F}^{+}-k_{F}^{-}\right)=\frac{2 m \gamma(\phi)}{\hbar^{2}}$ was used. Because $\left(k_{F}^{n}-k_{F}^{-n}\right)=n\left(k_{F}^{+}\right.$ $-k_{F}^{-}$), Eq. (25) becomes

$$
\sigma_{x y}^{s_{0}}=\frac{e}{16 \pi^{2}} \sum_{n} \oint_{C} d \mathbf{l} \cdot n\left[\mathbf{k} \times \mathbf{A}_{n}\right]_{z} \mathbf{a}_{0},
$$

where $\mathbf{A}_{n}=n \mathbf{A}$ with $n= \pm 1$. Using the definition of Berry phase in Eq. (16), the spin Hall conductivity can be written as

$$
\sigma_{x y}^{s_{0}}=\frac{e}{16 \pi^{2}} \sum_{n} \oint_{C} d \mathbf{l} \cdot\left(n \mathbf{A}_{n}\right)=\frac{e}{16 \pi^{2}} \sum_{n} n \Phi_{n},
$$

where $\Phi_{n}=n \Phi$ with $n= \pm 1$, in agreement with Ref. 23. In other words, the spin Hall conductivity can be written as a line integral of the Berry vector potential or a surface integral of the Berry curvature. Note that Eq. (27) holds irrespective of the choice of the eigenstates.

For the OAM Hall conductivity, similarly, Eq. (20) can be rewritten as

$$
\sigma_{x y}^{o_{0}}=\frac{e}{8 \pi^{2}} \sum_{n} \oint_{C} d \mathbf{l} \cdot\left[\mathbf{k} \times \mathbf{A}_{n}\right]_{z}^{2} \mathbf{a}_{0} .
$$

This expression has also been given in Ref. 33, where [ $\hbar \mathbf{k}$ $\times \mathbf{A}]_{z}=-\hbar\left(\lambda^{2}-\beta^{2}\right) / 2 \gamma^{2}(\phi)$ was regarded as the OAM of the eigenstates in the absence of any applied electric field. We believe that this interpretation is inappropriate because the expectation value of the conventional OAM operator depends on the choice of bases, as will be shown in the next section. In the next section we also argue that before an electric field is applied, the OAM of the eigenstates is zero. Finally, we also find that the ratio of the spin to OAM Hall conductivity is $\frac{\sigma_{x y}^{s_{0}}}{\sigma_{x y}^{\partial_{0}}}=\left.2[\mathbf{k} \times \mathbf{A}]_{z}\right|_{\phi=m \pi}$ where $m$ is an integer.

\section{E. Origin of the orbital angular momentum Hall current}

All electrons have an intrinsic spin of $\frac{1}{2}$. Before an inplane electric field is applied, the spins of the electrons are all aligned in the $x y$ plane, as shown in Figs. 1(a) and 1(b). When an in-plane electric field is applied, the SO coupling gives rise to not only a spin transverse force on a moving electron $^{34,35}$ but also an effective SO magnetic torque. The Rashba-Dresselhaus Hamiltonian can be written as $\left[H_{R}(\mathbf{k})\right.$ $\left.+H_{D}(\mathbf{k})\right]=\vec{\sigma} \cdot \mathbf{B}_{\text {eff }}$ where $\mathbf{B}_{\text {eff }} \equiv \lambda\left(\mathbf{k} \times \hat{e}_{z}\right)-\beta\left(k_{x} \hat{e}_{x}-k_{y} \hat{e}_{y}\right)$ is the effective SO magnetic field. The dynamics of the $\mathrm{z}$ component of spin can be derived from the Heisenberg equation of motion and we obtain $d \sigma_{z}(t) / d t=\frac{1}{i \hbar}\left[\sigma_{z}, \frac{p^{2}}{2 m}+H_{D}+H_{R}+e E y\right]$ $=\frac{1}{i \hbar}\left[\sigma_{z}, H_{D}+H_{R}\right]=\frac{1}{i \hbar}\left[\sigma_{z}, \sigma_{i}\right] B_{\text {eff. Using the commutation rela- }}^{i}$ tion of spin matrix $\left[\sigma_{i}, \sigma_{j}\right]=2 i \epsilon_{i j k} \sigma_{k}$, we have $\left[d s_{z}(t) / d t\right]$ $=-\frac{2}{\hbar}\left[\vec{s}(t) \times \mathbf{B}_{\text {eff }}\right]_{z}$, where $\vec{s}=\frac{\hbar}{2} \vec{\sigma}$. From the equation of motion of electron in $k$ space, we also have $\delta k_{y} \equiv\left[k_{y}\left(t_{0}\right)-k_{y}(t=0)\right]$ $=-\frac{e E}{\hbar} t_{0}$ and $\delta k_{x}=0$. Therefore, after a short time $t_{0}$, the Fermi surface (i.e., the circle for $n= \pm 1$ ) would move along the $-k_{y}$ direction with the distance $e E t_{0} / \hbar$. This implies that the variation of the effective SO magnetic field is $\delta \mathbf{B}_{\text {eff }}=\left(\lambda \hat{e}_{x}\right.$ $\left.+\beta \hat{e}_{y}\right) \delta k_{y}$. Consequently, each spin feels the effective SO magnetic torque $\vec{s}(0) \times \delta \mathbf{B}_{\text {eff }}$ and tilts out of the $x y$ plane. The quantum dynamical analysis of spin for the RashbaDresselhaus system has been given in Ref. 23 Here we use the quantum perturbation method to evaluate the response quantities.

Let us expand the wave function to first order of electric field, $|n \mathbf{k}\rangle^{\prime}=|n \mathbf{k}\rangle+|n \mathbf{k}\rangle^{(1)}$, where the perturbed wave function is

$$
|n \mathbf{k}\rangle^{(1)}=e E \sum_{n^{\prime}\left(n^{\prime} \neq n\right)} \frac{\left\langle n^{\prime} \mathbf{k}\left|i \partial / \partial k_{y}\right| n \mathbf{k}\right\rangle}{E_{n \mathbf{k}}-E_{n^{\prime} \mathbf{k}}}\left|n^{\prime} \mathbf{k}\right\rangle .
$$

The expectation value of the $z$ component of spin can be evaluated by ' $\left\langle n \mathbf{k}\left|s_{z}\right| n \mathbf{k}\right\rangle^{\prime}=\left\langle s_{z}\right\rangle_{0}+\delta\left\langle s_{z}\right\rangle$ where $\left\langle s_{z}\right\rangle_{0}$ $\equiv\left\langle n \mathbf{k}\left|s_{z}\right| n \mathbf{k}\right\rangle=0$ and the $z$ component of the spin in first order of electric field is given by $\delta\left\langle s_{z}\right\rangle \equiv 2 \operatorname{Re}\left\langle n \mathbf{k}\left|s_{z}\right| n \mathbf{k}\right\rangle^{(1)}$ $=-e E \hbar\left(\lambda^{2}-\beta^{2}\right) \cos \phi / 4 n k^{2} \gamma^{3}(\phi)$. Therefore, for $k_{x}>0$, the spins on the outer (inner) sheet tend to tilt down (up) and for $k_{x}<0$, the spins on the outer (inner) sheet tend to tilt up (down), as shown in the right-hand panel of Fig. 1(b). This results in transverse spin Hall currents with spin polarization in the $z$ direction.

Now consider the OAM $L_{z}=\hbar(\mathbf{x} \times \mathbf{k})_{z}$. The expectation value ' $\left\langle n \mathbf{k}\left|L_{z}\right| n \mathbf{k}\right\rangle^{\prime}=\left\langle L_{z}\right\rangle_{0}+\delta\left\langle L_{z}\right\rangle$ where $\left\langle L_{z}\right\rangle_{0} \equiv\left\langle n \mathbf{k}\left|L_{z}\right| n \mathbf{k}\right\rangle$ and $\delta\left\langle L_{z}\right\rangle=2 \operatorname{Re}\left\langle n \mathbf{k}\left|L_{z}\right| n \mathbf{k}\right\rangle^{(1)}$. In contrast to the spin case, before an electric field is applied, as argued below, all the eigenstates carry zero orbital angular momentum, i.e., $\left\langle L_{z}\right\rangle_{0}$ $=0$, as illustrated in the left-hand panel of Fig. 1(c). In the absence of the applied electric field, the diagonal matrix elements of the OAM depend on the choice of the eigenstates, though the off-diagonal matrix elements of the OAM do not. For example, if we choose the eigenstates of Eq. (5), together with the conventional position operator $\mathbf{x}=i \nabla_{\mathbf{k}},\left\langle L_{z}\right\rangle_{0}$ $=\langle n \mathbf{k}|\mathbf{x} \times \hbar \mathbf{k}| n \mathbf{k}\rangle=-\hbar\left(\lambda^{2}-\beta^{2}\right) / 2 \gamma^{2}(\phi)$, a value which has also been obtained in Ref. 33. On the other hand, if we use the eigenstates $|n \mathbf{k}\rangle=\frac{1}{\sqrt{2}}\left(\begin{array}{c}e^{-i \theta(\mathbf{k}) / 2} \\ i n e^{i \theta(\mathbf{k}) / 2}\end{array}\right),\left\langle L_{z}\right\rangle_{0}=0$. This is of course 
TABLE I. Total and decomposed spin (a) and orbital angular momentum (b) Hall conductivities of a two-dimensional electron system with Rashba-Dresselhuas spin-orbit coupling.

\begin{tabular}{|c|c|c|c|}
\hline & $\lambda^{2}>\beta^{2}$ & $\lambda^{2}=\beta^{2}$ & $\lambda^{2}<\beta^{2}$ \\
\hline \multicolumn{4}{|c|}{ (a) Spin Hall conductivity } \\
\hline$\sigma_{x y}^{s_{0}}$ & $e$ & 0 & $e$ \\
\hline$\sigma_{x y}^{\tau_{s}}$ & $e^{8 \pi}$ & 0 & $\overline{8 \pi}$ \\
\hline$\sigma_{x y}^{s}$ & $\overline{4 \pi}$ & 0 & $\begin{array}{r}4 \pi \\
\end{array}$ \\
\hline & $\overline{8 \pi}$ & & $\overline{8 \pi}$ \\
\hline \multicolumn{4}{|c|}{ (b) OAM Hall conductivity } \\
\hline$\sigma_{x y}^{o_{0}}$ & $\lambda^{2}+\beta^{2} e$ & 0 & $\lambda^{2}+\beta^{2} e$ \\
\hline$\sigma_{x y}^{\tau_{o}}$ & $\overline{\lambda^{2}-\beta_{\beta^{2}}^{2}} \overline{8 \pi}$ & 0 & $\overline{\mid \lambda_{\lambda^{2}+\beta^{2}}^{2}} \overline{8 \pi}$ \\
\hline$\sigma_{x y}^{o}$ & $\left|\lambda_{2}^{2}-\beta_{2}^{2}\right| \beta^{4 \pi}$ & 0 & $\left|\lambda_{2}^{2}-\beta_{\beta^{2}}^{2}\right| \stackrel{4 \pi}{e}$ \\
\hline & $\overline{\left|\lambda^{2}-\beta^{2}\right|} \overline{8 \pi}$ & & $\mid \overline{\lambda^{2}-\beta^{2} \mid} \overline{8 \pi}$ \\
\hline
\end{tabular}

unsatisfactory, and therefore, we propose to define a gauge invariant position operator $\mathbf{X}=i \nabla_{\mathbf{k}}+\mathbf{A}(\mathbf{k})$ to resolve the problem (see Appendix D). We can show that with this gauge invariant position operator $\mathbf{X},\left\langle L_{z}\right\rangle_{0}=0$, irrespective of the choice of the phase factor of the eigenstates. Therefore, we believe that all the eigenstates of the Rashba-Dresselhaus system carry zero OAM in the absence of applied electric fields. Importantly, the other quantities such as $\delta\left\langle s_{z}\right\rangle$ and $\delta\left\langle L_{z}\right\rangle$, which contain only the $n \neq n^{\prime}$ matrix elements, are independent of the choice of the phase factor and also the choice of the position operator.

As an in-plane electric field is applied, the dynamics of the z-component of the OAM can be obtained by means of Heisenberg equation of motion: $d L_{z}(t) / d t=\frac{d}{d t}(\hbar \mathbf{x} \times \mathbf{k})_{z}=$ $-\frac{2}{\hbar}\left[\vec{s}(t) \times \mathbf{B}_{\mathrm{eff}}^{\prime}\right]_{z}+(\mathbf{x} \times \mathbf{F})_{z}$ where $\mathbf{B}_{\mathrm{eff}}^{\prime}=\left.\mathbf{B}_{\mathrm{eff}}\right|_{\lambda \rightarrow-\lambda}$ and $\mathbf{F}=-e \mathbf{E}$ $=-e E \hat{e}_{y}$. The second term $(\mathbf{x} \times \mathbf{F})_{z}=\hbar \mathbf{x} \times \mathbf{k}$ is the classical external torque which causes the orbital motion of the electrons and depends on the choice of the origin of the coordinate system. The first term $-\frac{2}{\hbar}\left(\vec{s} \times \mathbf{B}_{\text {eff }}^{\prime}\right)_{z}$ is the effective SO magnetic torque. If we use the gauge invariant position operator, the classical torque $(\mathbf{x} \times \mathbf{F})_{z}$ does not contribute to the variation of the OAM in a short time $t_{0}$. We can show that $\frac{d}{d t}(\hbar \mathbf{X} \times \mathbf{k})_{z}=-\frac{2}{\hbar}\left[\vec{s}(t) \times \mathbf{B}_{\mathrm{eff}}^{\prime}\right]_{z}-X(t) e E+k_{y} \Omega_{z}$, where $\Omega_{z}=\nabla_{\mathbf{k}}$ $\times \mathbf{A} \sim \delta^{(2)}(\mathbf{k})$ and is zero because $\mathbf{k} \neq 0$. After a short time $t_{0}$, $\langle X(0)\rangle \delta k_{y}$ vanishes because $\langle X(0)\rangle=0$ and the $z$ component of the OAM for each eigenstate is induced by the effective SO magnetic torque and external torque only. In our calculations, we treat the potential $e E y$ as a perturbation and use again the quantum perturbation method instead of solving the Heisenberg equation of motion. We find that the $z$ component of the OAM is $\delta\left\langle L_{z}\right\rangle=e E \hbar\left(\lambda^{2}\right.$ $\left.-\beta^{2}\right)^{2} \cos \phi / 4 n k^{2} \gamma^{5}(\phi)=-\delta\left\langle s_{z}\right\rangle\left[\left(\lambda^{2}-\beta^{2}\right) / \gamma^{2}(\phi)\right]$, as illustrated in the right-hand panel of Fig. 1(c). Therefore, under the in-plane electric field along the $y$ axis, the electrons on the outer (inner) sheet with $k_{x}>0$ drift toward the $x$ axis, carrying the downward (upward) tilted spinors as well as finite positive (negative) OAM $\delta\left\langle L_{z}\right\rangle$, and the electrons on the outer (inner) sheet with $k_{x}<0$ drift toward the $x$ axis, carrying the upward (downward) tilted spinors as well as finite negative (positive) OAM $\delta\left\langle L_{z}\right\rangle$. This gives rise to the OAM Hall current. It is interesting to note that in the pure Rashba system $\left[\beta=0\right.$ and $\left.\left(\lambda^{2}-\beta^{2}\right) / \gamma^{2}(\phi)=1\right]$, the $\delta\left\langle s_{z}\right\rangle$ and $\delta\left\langle L_{z}\right\rangle$ have the same magnitude but the opposite signs and hence cancel each other exactly. This is due to the fact that the $z$ component of the total angular momentum is conserved in pure Rashba system.

\section{SYSTEMS WITH BOTH RASHBA AND DRESSELHAUS SPIN-ORBIT COUPLINGS}

The calculated total and decomposed spin and OAM Hall conductivities are summarized in Table I. The total spin and OAM Hall conductivities are displayed as a function of the ratio $|\lambda / \beta|$ in Fig. 2. The total angular momentum Hall conductivity $\left(\sigma_{x y}^{s_{0}}+\sigma_{x y}^{\tau_{s}}+\sigma_{x y}^{o_{0}}+\sigma_{x y}^{\tau_{o}}\right)$ is (see Table I)

$$
\sigma_{x y}=\left\{\begin{array}{cc}
-\frac{\beta^{2}}{\left|\lambda^{2}-\beta^{2}\right|} \frac{e}{4 \pi}, & \lambda^{2} \neq \beta^{2}, \\
0, & \lambda^{2}=\beta^{2} .
\end{array}\right.
$$

It is clear that in 2DEG systems with the Rashba-Dresselhaus SO coupling, the total angular momentum Hall conductivity is in general not zero, except that $\lambda^{2}=\beta^{2}$ or $\beta=0$ (pure

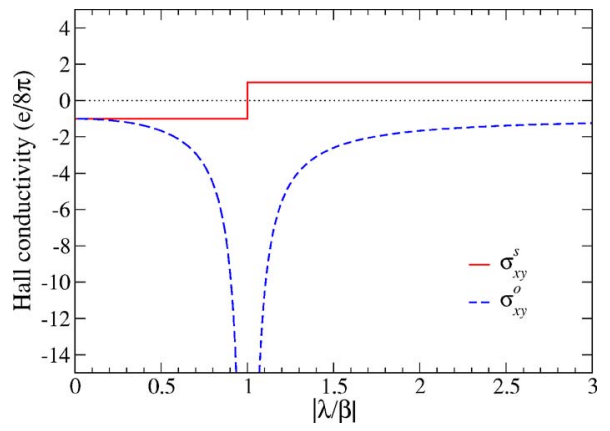

FIG. 2. (Color online) Spin $\left(\sigma_{x y}^{s}\right.$, solid line) and orbital angular momentum $\left(\sigma_{x y}^{o}\right.$, dashed line) Hall conductivities versus the strength ratio $|\lambda / \beta|$ of the Rashba $(\lambda)$ to Dresselhaus $(\beta)$ spin-orbit coupling. 
Rashba SO coupling). The sign of the total angular momentum Hall conductivity is always negative, and, as will be discussed shortly, this is because the Hall conductivity is dominated by the negative OAM Hall conductivity. Furthermore, when the two SO coupling strengths are comparable, the total angular momentum Hall conductivity is very large, suggesting the interesting possibility of tuning the angular momentum Hall effect by varying the Rashba SO coupling strength. This large total Hall conductivity results from the large OAM Hall conductivity in both the conventional and present definitions of spin current (see Table I). The conventional OAM Hall conductivity is the same as that given in Ref. 33. This singularly large OAM conductivity in the region that $|\lambda / \beta|$ approaches to unity, could lead to spontaneous magnetization, as suggested in Ref. 33. Nevertheless, we believe that this singular behavior of the OAM conductivity near $\lambda \sim \beta$ is unphysical and is perhaps due to the neglect of the disorder in the present calculations. The disorder due to, e.g., impurity scattering and electron-electron interaction is known to have pronounced effects on the spin Hall effect. ${ }^{9-11}$ The infinite large OAM conductivity is expected to be suppressed by the disorder effects in real 2DEG systems. Nonetheless, further calculations taking into account the disorder effects are beyond the scope of the present paper.

Shen $^{23}$ recently pointed out that in the 2DEG systems with both Rashba and Dresselhaus couplings, the spin current along the $z$ direction is antisymmetric with respect to a unitary transformation, $\sigma_{x} \rightarrow \sigma_{y} ; \sigma_{y} \rightarrow \sigma_{x} ; \sigma_{z} \rightarrow-\sigma_{z}$. This antisymmetry makes the conventional spin Hall conductivity change sign at $\lambda^{2}=\beta^{2}$. It is interesting to note that the spin torque Hall conductivity and hence the total spin Hall conductivity also change sign at $\lambda^{2}=\beta^{2}$ when one moves from the region where Dresselhaus coupling dominates the region where Rashba coupling dominates (Table I and Fig. 2). This shows that our calculated spin torque and total spin Hall conductivities also obey the requirement of this antisymmetry. We also note that as for the case of pure Rashba coupling, ${ }^{16}$ the spin torque Hall conductivity is 2 times as large as the conventional spin Hall conductivity but has an opposite sign (Table I), giving rise to the result that the conserved spin Hall conductivity has the same size but opposite sign to the conventional spin Hall conductivity.

On the other hand, all the (total, torque and conventional) OAM Hall conductivities do not change sign at $\lambda^{2}=\beta^{2}$, as shown in Table I and Fig. 2. As for the case of spin Hall effect, the torque OAM Hall conductivity is 2 times as large as the conventional OAM Hall conductivity but has an opposite sign, resulting in that the effective OAM Hall conductivity has the same size but opposite sign to the conventional OAM Hall conductivity (Table I). The conventional OAM Hall conductivity has been reported in Ref. 33, and our result is consistent with this previous calculation.

\section{SYSTEMS WITH EITHER PURE RASHBA TERM OR PURE DRESSELHAUS TERM}

\section{A. Pure Rashba spin-orbit coupling}

In the case of $\beta=0$ and $\lambda \neq 0$, the Hamiltonian reduces to the pure Rashba Hamiltonian. The spin torque operator becomes

$$
\tau_{s}^{R} \equiv \frac{1}{i \hbar}\left[s_{z}, \frac{p^{2}}{2 m}+H_{R}\right]
$$

and the $y$ component of the velocity operator is

$$
v_{y}^{R}=\frac{p_{y}}{m}+\frac{\lambda}{\hbar} \sigma_{x} .
$$

The torque correction term for the spin Hall conductivity is $e / 4 \pi$, and for the OAM Hall conductivity is $-e / 4 \pi$, which exactly cancel each other. This result is confirmed by the relation $\left[L_{z}, H_{R}\right]=-\left[s_{z}, H_{R}\right]$ that is present in the Rashba Hamiltonian. Including the torque correction term, we get an effective spin Hall conductivity of $e / 8 \pi$, and an effective OAM Hall conductivity of $-e / 8 \pi$. This implies that when the torque correction term is taken into account, the spin Hall current is still exactly canceled by the accompanied OAM Hall current and there is no total angular momentum current in the pure Rashba system. This interesting observation has been reported before in Ref. 7 in the context of the conventional definition of the spin and OAM currents. This may be expected because $\left[s_{z}+L_{z}, H_{R}\right]=0$ and the conservation of the total angular momentum therefore must be obeyed no matter what definitions of the effective angular momentum currents one adopts.

Nonetheless, we want to point out here that the spin Hall effect in the pure Rashba Hamiltonian can still manifest itself and be detected in several ways, even though the total angular momentum Hall current is zero. It is well known that the magnetic dipole moment associated with the spin of an electron is $\mu_{s}=-2 \mathrm{~s} \mu_{B}$ and the one associated with the OAM of an electron is $\mu_{o}=-\mathbf{L} \mu_{B}$ (see, e.g., Ref. 36). Consequently, although the total angular momentum $\left(s_{z}+L_{z}\right)$ current is zero, the total magnetization $\left[\left(\mu_{s}^{z}+\mu_{o}^{z}\right)=-\left(2 s_{z}+L_{z}\right) \mu_{B}\right]$ current would not be zero and will give rise to finite magnetization at the edge of the sample. Therefore, the spin Hall effect in the pure Rashba Hamiltonian can in principle be probed in at least two ways. As noted in Ref. 7, one is by measuring the electric field induced by the nonzero magnetization current ${ }^{37}$ and the other is the magnetization at the sample edges. Recently, it is suggested in Ref. 11 that spin current can be detected by measuring time-dependent magnetization precession.

\section{B. Pure Dresselhaus spin-orbit coupling}

In the case of $\lambda=0$ and $\beta \neq 0$, the Hamiltonian reduces to the pure Dresselhaus Hamiltonian. In this case, the total, torque correction and conventional spin and OAM Hall conductivities have the same signs (Table I). For example, the total spin Hall conductivity is $-e / 8 \pi$, being the same as the total OAM Hall conductivity, thereby giving rise to a total angular momentum Hall conductivity of $-e / 4 \pi$. In the Dresselhaus Hamiltonian, the total angular momentum is not conserved, i.e., $\left[s_{z}+L_{z}, H_{D}\right]=-2 i \beta\left(\sigma_{x} p_{y}+\sigma_{y} p_{x}\right) \neq 0$. Instead, we find $\left[s_{z}, H_{D}\right]=\left[L_{z}, H_{D}\right]$ or $\left[s_{z}-L_{z}, H_{D}\right]=0$. Therefore, the spin and OAM Hall conductivities would add up rather than cancel each other, in contrast to the Rashba Hamiltonian.

We notice that the spin Hall conductivity in the clean limit is constant in either pure Rashba system, or pure Dresselhaus 
system, or the mixture Rashba-Dresselhaus system [see (a) in Table I]. Interestingly, in Refs. 10 and 11, it is shown that the constant spin Hall conductivity in the pure Rashba system would result in an unphysical growth of the magnetization with time. This is due to the fact that the conventionally defined spin Hall current in the pure Rashba system turns out to be proportional to the time derivative of the magnetizaion of the system. This linear relation between the conventional spin Hall current and the time derivative of the magnetization should also exist in the pure Dresselhaus system because the two systems are related by the unitary transformation: $\sigma_{x} \rightarrow \sigma_{y}$ and $\sigma_{y} \rightarrow \sigma_{x}$, as mentioned in Sec. III. Indeed, we find that the conventional spin current in the pure Dresselhaus system is $j_{x}^{s_{z}}=\frac{\hbar}{4}\left\{v_{x}, \sigma_{z}\right\}=\frac{\hbar}{2 m} \sigma_{z} p_{x}$, where the relation $\left\{\sigma_{i}, \sigma_{j}\right\}=2 \delta_{i j}$ was used. Furthermore, it can be shown that the spin precession and conventional spin current satisfy the relations $\frac{d\left\langle\sigma_{y}(t)\right\rangle}{d t}=\frac{2 \beta}{\hbar^{2}}\left\langle p_{x} \sigma_{z}\right\rangle(t)=\frac{4 m \beta}{\hbar^{3}}\left\langle j_{x}^{s_{z}}(t)\right\rangle$ and $\frac{d\left\langle\sigma_{x}(t)\right\rangle}{d t}=\frac{4 m \beta}{\hbar^{3}}\left\langle j_{y}^{s_{z}}(t)\right\rangle$. Therefore, the constant conventional spin current in the clean limit in the pure Dresselhaus system would also lead to the unphysical consequence that both $\left\langle\sigma_{y}(t)\right\rangle$ and $\left\langle\sigma_{x}(t)\right\rangle$ depend linearly on time, resulting in an infinite growth of the magnetization. In contrast, because the conserved spin current satisfies the continuity equation [Eq. (1)], we find no such relation that the conserved spin current is proportional to the time derivative of the spin operator, thereby, free from the artifact discussed above, as also pointed out in Ref. 38 .

\section{CONCLUSIONS}

In conclusion, we have calculated the spin Hall coductivity in the Rashba-Dresselhaus Hamiltonian with the spin torque correction in the absence of disorder, and find it to have the same magnitude but an opposite sign to the result reported before. ${ }^{4,23}$ The spin Hall conductivity in the absence of disorder is still a constant in the pure Dresselhaus (or Rashba) system even when the torque correction is considered. We also introduce the conserved effective OAM current and find that in general, the OAM Hall effect does not cancel the spin Hall effect in the 2DEG with the RashbaDresselhaus spin-orbit coupling. The OAM Hall conductivity depends significantly on the strength ratio of the Rashba to Dresselhaus spin-orbit coupling, suggesting that one can manipulate the total Hall current through tuning the Rashba coupling by a gate voltage. ${ }^{39,40} \mathrm{We}$ argue that in a pure Rashba system, though the spin Hall conductivity is exactly cancelled by the OAM Hall conductivity due to the angular momentum conservation, the spin Hall effect still manifest itself as nonzero magnetization Hall current and finite magnetization at the sample edges because the magnetic dipole moment associated with the spin of an electron 2 times as large as that of the OAM. We show that the spin and OAM Hall conductivities have a simple relation to the Berry vector (or gauge) potential. We also calculate the electric fieldinduced OAM and discuss the origin of the OAM Hall current.

\section{ACKNOWLEDGMENTS}

The authors thank Ming-Che Chang and Qian Niu for stimulating discussions. The authors gratefully acknowledge financial support from the National Science Council of Taiwan.

\section{APPENDIX A: THE CONTINUITY EQUATION FOR THE EFFECTIVE SPIN CURRENT}

In this appendix, we derive the continuity equation for the effective spin current. A similar derivation can also be found in Ref. 15 The definition of spin density is $\mathcal{S}_{z}=\Psi^{\dagger} s_{z} \Psi$, where $s_{z}=\frac{\hbar}{2} \sigma_{z}$. Using $i \hbar \frac{\partial}{\partial t} \Psi=H \Psi$ and $H=H_{0}+e \mathbf{E} \cdot \mathbf{x}$, we obtain the following equation:

$$
\begin{aligned}
\frac{\partial}{\partial t}\left[\Psi^{\dagger} \sigma_{z} \Psi\right]= & \frac{1}{i \hbar}\left[\Psi^{\dagger} \sigma_{z} H_{0} \Psi-\left(H_{0} \Psi\right)^{\dagger} \sigma_{z} \Psi\right] \\
= & \frac{1}{i \hbar}\left\{\left[\Psi^{\dagger} \sigma_{z} \frac{p^{2}}{2 m} \Psi-\left(\frac{p^{2}}{2 m} \Psi\right)^{\dagger} \sigma_{z} \Psi\right]\right. \\
& +\left[\Psi^{\dagger} \sigma_{z} H_{R} \Psi-\left(H_{R} \Psi\right)^{\dagger} \sigma_{z} \Psi\right]+\left[\Psi^{\dagger} \sigma_{z} H_{D} \Psi\right. \\
& \left.\left.-\left(H_{D} \Psi\right)^{\dagger} \sigma_{z} \Psi\right]\right\},
\end{aligned}
$$

where we have used $\left[s_{z}, \mathbf{x}\right]=0$. First, we combine the following two equations:

$$
\begin{aligned}
& \frac{\partial}{\partial x_{i}}\left(\Psi^{\dagger} \frac{\partial}{\partial x_{i}} \sigma_{z} \Psi\right)=\left(\frac{\partial \Psi^{\dagger}}{\partial x_{i}}\right) \frac{\partial}{\partial x_{i}} \sigma_{z} \Psi+\Psi^{\dagger} \frac{\partial}{\partial x_{i}} \frac{\partial}{\partial x_{i}} \sigma_{z} \Psi, \\
& \frac{\partial}{\partial x_{i}}\left(\frac{\partial \Psi^{\dagger}}{\partial x_{i}} \sigma_{z} \Psi\right)=\left(\frac{\partial}{\partial x_{i}} \frac{\partial}{\partial x_{i}} \Psi^{\dagger}\right) \sigma_{z} \Psi+\frac{\partial \Psi^{\dagger}}{\partial x_{i}}\left(\frac{\partial}{\partial x_{i}} \sigma_{z} \Psi\right) .
\end{aligned}
$$

Using the definition of momentum operator $\mathbf{p}=-i \hbar \nabla$ and $\left(\Psi^{\dagger} \mathbf{p} \sigma_{z} \Psi\right)^{\dagger}=-\left(\mathbf{p} \Psi^{\dagger}\right) \sigma_{z} \Psi$, we obtain

$$
\mathbf{p} \cdot\left[2 \operatorname{Re}\left(\Psi^{\dagger} \mathbf{p} \sigma_{z} \Psi\right)\right]=\left[\Psi^{\dagger} p^{2} \sigma_{z} \Psi-\left(p^{2} \Psi^{\dagger}\right) \sigma_{z} \Psi\right] .
$$

Next, consider the term $\left[\Psi^{\dagger} \sigma_{z} H_{R} \Psi-\left(H_{R} \Psi\right)^{\dagger} \sigma_{z} \Psi\right]$ which contains only the Rashba Hamiltonian. We obtain the following equations:

$$
\begin{aligned}
{\left[\Psi^{\dagger} \sigma_{z} H_{R} \Psi-\left(H_{R} \Psi\right)^{\dagger} \sigma_{z} \Psi\right]=} & \frac{2 i \lambda}{\hbar} \Psi^{\dagger} \mathbf{p} \cdot \vec{\sigma} \Psi \\
& -\frac{\lambda}{\hbar} \mathbf{p} \cdot\left[\Psi^{\dagger} \sigma_{z}\left(\vec{\sigma} \times \hat{e}_{z}\right) \Psi\right]
\end{aligned}
$$

and

$$
\begin{aligned}
{\left[\Psi^{\dagger} \sigma_{z} H_{R} \Psi-\left(H_{R} \Psi\right)^{\dagger} \sigma_{z} \Psi\right]=} & -\left[\Psi^{\dagger} \sigma_{z} H_{R} \Psi-\left(H_{R} \Psi\right)^{\dagger} \sigma_{z} \Psi\right]^{\dagger} \\
= & \frac{2 i \lambda}{\hbar}\left[\Psi^{\dagger} \mathbf{p} \cdot \vec{\sigma} \Psi\right]^{\dagger} \\
& -\frac{\lambda}{\hbar} \mathbf{p} \cdot\left[\Psi^{\dagger} \sigma_{z}\left(\vec{\sigma} \times \hat{e}_{z}\right) \Psi\right]^{\dagger}
\end{aligned}
$$

We combine Eq. (A3) and Eq. (A4), 


$$
\begin{aligned}
{\left[\Psi^{\dagger} \sigma_{z} H_{R} \Psi-\left(H_{R} \Psi\right)^{\dagger} \sigma_{z} \Psi\right]=} & \frac{2 i \lambda}{\hbar} \operatorname{Re}\left[\Psi^{\dagger}(\mathbf{p} \cdot \vec{\sigma}) \Psi\right] \\
& -\frac{\lambda}{\hbar} \mathbf{p} \cdot \operatorname{Re}\left[\Psi^{\dagger} \sigma_{z}\left(\vec{\sigma} \times \hat{e}_{z}\right) \Psi\right]
\end{aligned}
$$

Now consider the term $\left[\Psi^{\dagger} \sigma_{z} H_{D} \Psi-\left(H_{D} \Psi\right)^{\dagger} \sigma_{z} \Psi\right]$ which contains only the Dresselhaus Hamiltonian. From the second term $\left(H_{D} \Psi\right)^{\dagger} \sigma_{z} \Psi$, we obtain

$$
\begin{aligned}
\left(H_{D} \Psi\right)^{\dagger} \sigma_{z} \Psi= & -\frac{\beta}{\hbar}\left(-p_{x} \Psi^{\dagger} \sigma_{x}+p_{y} \Psi^{\dagger} \sigma_{y}\right) \sigma_{z} \Psi \\
= & \frac{\beta}{\hbar} p_{x}\left(\Psi^{\dagger} \sigma_{x} \sigma_{z} \Psi\right)-\frac{\beta}{\hbar} \Psi^{\dagger} p_{x} \sigma_{x} \sigma_{z} \Psi \\
& -\frac{\beta}{\hbar} p_{y}\left(\Psi^{\dagger} \sigma_{y} \sigma_{z} \Psi\right)+\frac{\beta}{\hbar} \Psi^{\dagger} p_{y} \sigma_{y} \sigma_{z} \Psi \\
= & -\frac{\beta}{\hbar}\left[p_{x}\left(\Psi^{\dagger} \sigma_{z} \sigma_{x} \Psi\right)-p_{y}\left(\Psi^{\dagger} \sigma_{z} \sigma_{y} \Psi\right)\right]
\end{aligned}
$$

$$
-\frac{\beta}{\hbar} \Psi^{\dagger}\left(p_{x} \sigma_{x}-p_{y} \sigma_{y}\right) \sigma_{z} \Psi
$$

and then

$$
\begin{aligned}
{\left[\Psi^{\dagger} \sigma_{z} H_{D} \Psi-\left(H_{D} \Psi\right)^{\dagger} \sigma_{z} \Psi\right]=} & \frac{-2 i \beta}{\hbar} \Psi^{\dagger}\left(p_{x} \sigma_{y}+p_{y} \sigma_{x}\right) \Psi \\
& +\frac{\beta}{\hbar}\left[p_{x}\left(\Psi^{\dagger} \sigma_{z} \sigma_{x} \Psi\right)\right. \\
& \left.-p_{y}\left(\Psi^{\dagger} \sigma_{z} \sigma_{y} \Psi\right)\right] \\
= & \frac{-2 i \beta}{\hbar} \operatorname{Re}\left[\Psi^{\dagger}\left(p_{x} \sigma_{y}+p_{y} \sigma_{x}\right) \Psi\right] \\
& +\frac{\beta}{\hbar}\left[p_{x} \operatorname{Re}\left(\Psi^{\dagger} \sigma_{z} \sigma_{x} \Psi\right)\right. \\
& \left.-p_{y} \operatorname{Re}\left(\Psi^{\dagger} \sigma_{z} \sigma_{y} \Psi\right)\right],
\end{aligned}
$$

where we have used the relation $\left[\Psi^{\dagger} \sigma_{z} H_{D} \Psi-\left(H_{D} \Psi\right)^{\dagger} \sigma_{z} \Psi\right]$ $=-\left[\Psi^{\dagger} \sigma_{z} H_{D} \Psi-\left(H_{D} \Psi\right)^{\dagger} \sigma_{z} \Psi\right]^{\dagger}$. Substituting Eqs. (A2), (A5), and (A7) into (A1), we obtain

$$
\frac{\partial}{\partial t}\left(\Psi^{\dagger} \sigma_{z} \Psi\right)=-\nabla \operatorname{Re}\left[\Psi^{\dagger} \sigma_{z}\left(\frac{\mathbf{p}}{m}-\frac{\lambda}{\hbar} \vec{\sigma} \times \hat{e}_{z}-\frac{\beta}{\hbar}\left(\sigma_{x} \hat{e}_{x}-\sigma_{y} \hat{e}_{y}\right)\right) \Psi\right]+\frac{2 \lambda}{\hbar^{2}} \operatorname{Re}\left[\Psi^{\dagger}(\mathbf{p} \cdot \vec{\sigma}) \Psi\right]-\frac{2 \beta}{\hbar^{2}} \operatorname{Re}\left[\Psi^{\dagger}\left(p_{x} \sigma_{y}+p_{y} \sigma_{x}\right) \Psi\right]
$$

Consider the commutator $\left[\sigma_{z}, H_{0}\right]$, and we have

$$
\frac{1}{i \hbar}\left[\sigma_{z}, \frac{p^{2}}{2 m}+H_{R}+H_{D}\right]=\frac{2 \lambda}{\hbar^{2}} \mathbf{p} \cdot \vec{\sigma}+\frac{-2 \beta}{\hbar^{2}}\left(p_{x} \sigma_{y}+p_{y} \sigma_{x}\right) .
$$

The velocity operator in the Rashba-Dresselhaus system satisfy the following relation:

$$
\mathbf{v}=\frac{1}{i \hbar}[\mathbf{x}, H]=\frac{1}{i \hbar}\left[\mathbf{x}, H_{0}\right]=\frac{\mathbf{p}}{m}-\frac{\lambda}{\hbar}\left(\vec{\sigma} \times \hat{e}_{z}\right)-\frac{\beta}{\hbar}\left(\sigma_{x} \hat{e}_{x}-\sigma_{y} \hat{e}_{y}\right) .
$$

Finally, substituting Eq. (A10) and Eq. (A9) into Eq. (A8), we obtain the continuity equation of the effective spin current

$$
\frac{\partial}{\partial t}\left[\Psi^{\dagger} s_{z} \Psi\right]=-\nabla \operatorname{Re}\left[\Psi^{\dagger} \mathbf{j}^{s_{z}} \Psi\right]+\operatorname{Re}\left[\Psi^{\dagger} \tau_{s} \Psi\right]
$$

where we have used $s_{z}=\frac{\hbar}{2} \sigma_{z}, \mathbf{j}^{s_{z}}=\frac{1}{2}\left\{\mathbf{v}, s_{z}\right\}$ and $\tau_{s}=\frac{1}{i \hbar}\left[s_{z}, H_{0}\right]$. With the definition of spin density $\mathcal{S}_{z}=\Psi^{\dagger} s_{z} \Psi$, spin current $\mathbf{J}_{s}=\operatorname{Re}\left[\Psi^{\dagger} \mathbf{j}^{s} \Psi\right]$ and spin torque $\mathcal{T}_{z}^{s}=\operatorname{Re}\left[\Psi^{\dagger} \tau_{s} \Psi\right]$, Eq. (A11) can be rewritten as

$$
\frac{\partial \mathcal{S}_{z}}{\partial t}+\nabla \cdot \mathbf{J}_{s}=\mathcal{T}_{z}^{s}
$$

which is in agreement with Ref. 16.

\section{APPENDIX B: THE CONTINUITY EQUATION FOR THE EFFECTIVE OAM CURRENT}

In this appendix, we derive the continuity equation for the effective OAM current. Let us consider the $z$ component of the OAM density $\mathcal{L}_{z} \equiv \Psi^{\dagger} L_{z} \Psi$ and its partial time-derivative $i \hbar \frac{\partial}{\partial t} \mathcal{L}_{z}$. Applying $i \hbar \frac{\partial}{\partial t} \Psi=H \Psi$ where $H=H_{0}+e \mathbf{E} \cdot \mathbf{x}$, we have

$$
\begin{aligned}
i \hbar \frac{\partial}{\partial t}\left(\Psi^{\dagger} L_{z} \Psi\right)= & \Psi^{\dagger} L_{z} H_{0} \Psi-\left(H_{0} \Psi\right)^{\dagger} L_{z} \Psi+\Psi^{\dagger}\left[L_{z}, e \mathbf{E} \cdot \mathbf{x}\right] \Psi \\
= & \Psi^{\dagger} L_{z}\left(\frac{p^{2}}{2 m}+H_{R}+H_{D}\right) \Psi \\
& -\left[\left(\frac{p^{2}}{2 m}+H_{R}+H_{D}\right) \Psi\right]^{\dagger} \\
& \times L_{z} \Psi+\Psi^{\dagger}\left[L_{z}, e \mathbf{E} \cdot \mathbf{x}\right] \Psi
\end{aligned}
$$

First, using the commutation relations $\left[\mathbf{p}, L_{z}\right]=i \hbar \hat{e}_{z} \times \mathbf{p}$ and $\left[L_{z}, p^{2}\right]=0$, we can show that 


$$
\begin{aligned}
\left\{\Psi^{\dagger} L_{z} p^{2} \Psi-\left(p^{2} \Psi^{\dagger}\right) L_{z} \Psi\right\}= & \mathbf{p} \cdot\left[\Psi^{\dagger} \mathbf{p} L_{z} \Psi-\left(\mathbf{p} \Psi^{\dagger}\right)\left(L_{z} \Psi\right)\right] \\
= & \mathbf{p} \cdot\left[\Psi^{\dagger}\left\{\mathbf{p}, L_{z}\right\} \Psi\right]-p^{2}\left(\Psi^{\dagger} L_{z} \Psi\right) \\
& +\mathbf{p} \cdot\left(\Psi^{\dagger} i \hbar \hat{e}_{z} \times \mathbf{p} \Psi\right) .
\end{aligned}
$$

For the Rashba Hamiltonian $H_{R}$, we have

$$
\begin{aligned}
\left(H_{R} \Psi\right)^{\dagger} L_{z} \Psi= & \frac{\lambda}{\hbar}\left(-p_{y} \Psi^{\dagger} \sigma_{x}+p_{x} \Psi^{\dagger} \sigma_{y}\right) L_{z} \Psi \\
= & \frac{\lambda}{\hbar} \mathbf{p} \cdot\left[\Psi^{\dagger}\left(\vec{\sigma} \times \hat{e}_{z}\right) L_{z} \Psi\right] \\
& +\frac{\lambda}{\hbar} \Psi^{\dagger}\left(p_{y} \sigma_{x}-p_{x} \sigma_{y}\right) L_{z} \Psi
\end{aligned}
$$

and

$$
\begin{aligned}
\left\{\Psi^{\dagger} L_{z} H_{R} \Psi-\left(H_{R} \Psi\right)^{\dagger} L_{z} \Psi\right\}= & \Psi^{\dagger}\left[L_{z}, H_{R}\right] \Psi \\
& -\frac{\lambda}{\hbar} \mathbf{p} \cdot\left[\Psi^{\dagger}\left(\vec{\sigma} \hat{e}_{z}\right) L_{z} \Psi\right] .
\end{aligned}
$$

For the Dresselhaus Hamiltonian $H_{D}$, we obtain

$$
\begin{aligned}
\left\{\Psi^{\dagger} L_{z} H_{D} \Psi-\left(H_{D} \Psi\right)^{\dagger} L_{z} \Psi\right\}= & \Psi^{\dagger}\left[L_{z}, H_{D}\right] \Psi-\frac{\beta}{\hbar} \mathbf{p} \cdot\left[\Psi ^ { \dagger } \left(\sigma_{x} \hat{e}_{x}\right.\right. \\
& \left.\left.-\sigma_{y} \hat{e}_{y}\right) L_{z} \Psi\right] .
\end{aligned}
$$

Substituting Eqs. (B2)-(B4) into Eq. (B1), we have

$$
\begin{aligned}
i \hbar \frac{\partial}{\partial t}\left(\Psi^{\dagger} L_{z} \Psi\right)= & \mathbf{p} \cdot\left[\Psi^{\dagger}\left\{\frac{\mathbf{p}}{2 m}, L_{z}\right\} \Psi+\Psi^{\dagger}\left[L_{z}, e \mathbf{E} \cdot \mathbf{x}\right] \Psi\right. \\
& -\frac{\lambda}{\hbar} \Psi^{\dagger}\left(\vec{\sigma} \times \hat{e}_{z}\right) L_{z} \Psi \\
& \left.-\frac{\beta}{\hbar} \Psi^{\dagger}\left(\sigma_{x} \hat{e}_{x}-\sigma_{y} \hat{e}_{y}\right) L_{z} \Psi\right] \\
& +\Psi^{\dagger}\left[L_{z}, H_{R}+H_{D}\right] \Psi \\
& -\frac{1}{2 m} p^{2}\left(\Psi^{\dagger} L_{z} \Psi\right)+\frac{1}{2 m} \mathbf{p} \cdot\left(\Psi^{\dagger} i \hbar \hat{e}_{z} \times \mathbf{p} \Psi\right) .
\end{aligned}
$$

Setting Eq. (A10) into Eq. (B5), we have

$$
\begin{aligned}
\frac{\partial}{\partial t}\left(\Psi^{\dagger} L_{z} \Psi\right)= & -\nabla \cdot\left[\Psi^{\dagger} \frac{1}{2}\left\{\mathbf{v}, L_{z}\right\} \Psi\right]+\Psi^{\dagger} \frac{1}{i \hbar}\left[L_{z}, e \mathbf{E} \cdot \mathbf{x}\right] \Psi \\
& +\Psi^{\dagger} \frac{1}{i \hbar}\left[L_{z}, H_{0}\right] \Psi-\frac{i \hbar}{2 m} \nabla^{2}\left(\Psi^{\dagger} L_{z} \Psi\right) \\
& -\mathbf{p} \cdot\left[\Psi^{\dagger} \hat{e}_{z} \times \mathbf{p} \Psi\right] .
\end{aligned}
$$

Finally, it can be shown that $\mathbf{p} \cdot\left(\Psi^{\dagger} \hat{e}_{z} \times \mathbf{p} \Psi\right)$ is purely imaginary, and $\Psi^{\dagger} L_{z} \Psi=\operatorname{Re}\left(\Psi^{\dagger} L_{z} \Psi\right)$ is real for both the eigenstates of the Rashba-Dresselhauls Hamiltonian and the Bloch states $\Psi_{\mathbf{k}}$. The $\mathcal{L}_{z}$ continuity equation becomes $\frac{\partial}{\partial t}\left(\Psi^{\dagger} L_{z} \Psi\right)=-\nabla \cdot \operatorname{Re}\left(\Psi^{\dagger} \mathbf{j}^{o_{0}} \Psi\right)+\operatorname{Re}\left(\Psi^{\dagger} \tau_{o} \Psi\right)+\operatorname{Re}\left(\Psi^{\dagger} \tau_{E} \Psi\right)$,

where $\mathbf{j}^{O_{0}}=\frac{1}{2}\left\{\mathbf{v}, L_{z}\right\}, \quad \tau_{o}=\frac{1}{i \hbar}\left[L_{z}, H_{0}\right], \quad$ and $\tau_{E}=\frac{1}{i \hbar}\left[L_{z}, e \mathbf{E} \cdot \mathbf{x}\right]$. With the OAM current density $\mathbf{J}_{o}=\operatorname{Re}\left(\Psi^{\dagger} \mathbf{j}^{{ }^{0}} \Psi\right)$, the OAM torque density $\mathcal{T}_{z}^{o}=\operatorname{Re}\left(\Psi^{\dagger} \tau_{o} \Psi\right)$ and the classical torque density $\mathcal{T}_{z}^{E}=\operatorname{Re}\left(\Psi^{\dagger} \tau_{E} \Psi\right)$, Eq. (B6) can be rewritten as

$$
\frac{\partial \mathcal{L}_{z}}{\partial t}+\nabla \cdot \mathbf{J}_{o}=\mathcal{T}_{z}^{o}+\mathcal{T}_{z}^{E},
$$

which is the OAM continuity equation.

The torque $\mathcal{T}_{z}^{E}=\operatorname{Re}\left(\Psi^{\dagger} \tau_{E} \Psi\right)$ has a classical analogue. It can be regarded as the rotational torque moment due to the force $e \mathbf{E}$ exerted on a particle located at the position $\mathbf{x}$ with respect to the origin of the coordinate system. This can be seen from the commutator $\left[L_{z}, e \mathbf{E} \cdot \mathbf{x}\right]=-i \hbar e(\mathbf{x} \times \mathbf{E})_{z}$, and

$$
\mathcal{T}_{z}^{E}=-\operatorname{Re}\left[\left(\Psi^{\dagger} \mathbf{x} \Psi\right) \times e \mathbf{E}\right]_{z} .
$$

From the space integral $\int d V \mathcal{T}_{z}^{E}=-\operatorname{Re}\left[\left(\int d V \Psi^{\dagger} \mathbf{x} \Psi\right) \times e \mathbf{E}\right]_{z}$, we obtain $\int d V \mathcal{T}_{z}^{E}=-\operatorname{Re}[\langle\Psi|\mathbf{x}| \Psi\rangle \times(e \mathbf{E})]_{z}$, where $\int d V \Psi \mathbf{x} \Psi$ $=\langle\Psi|\mathbf{x}| \Psi\rangle$. Expanding the $\langle\Psi|\mathbf{x}| \Psi\rangle$ in powers of electric field, we have $\langle\Psi|\mathbf{x}| \Psi\rangle=\left\langle\Psi_{0}|\mathbf{x}| \Psi_{0}\right\rangle+\langle\mathbf{x}\rangle^{(1)}+o\left(E^{2}\right)$, where $\left|\Psi_{0}\right\rangle$ satisfies the unperturbed wave equation $i \hbar \frac{\partial}{\partial t}\left|\Psi_{0}\right\rangle$ $=H_{0}\left|\Psi_{0}\right\rangle$. Therefore, we obtain $\int d V \mathcal{T}_{z}^{E}=-\operatorname{Re}\left[\left\langle\Psi_{0}|\mathbf{x}| \Psi_{0}\right\rangle\right.$ $\times(e \mathbf{E})]_{z}+o\left(E^{2}\right)$. However, we should demand that the expectation value of the OAM in the unperturbed system is zero. This would imply that the expectation value of the position operator must be zero in the absence of the external electric field, as discussed in Appendix D. Finally, we obtain that the OAM continuity equation

$$
\frac{\partial \mathcal{L}_{z}}{\partial t}+\nabla \cdot \mathbf{J}_{o}=\mathcal{T}_{z}^{o}
$$

which is valid in first order of the electric field.

\section{APPENDIX C: SPIN AND OAM TORQUE HALL CONDUCTIVITY}

In this appendix, we derive the spin and orbital angular momentum (OAM) torque Hall conductivities $\left(\sigma_{x y}^{\tau}\right)$ in the Rashba-Dresselhaus system. The conductivity in pure Rashba system can be obtained by setting $\beta=0$ in the $\lambda^{2}$ $>\beta^{2}$ condition. The total torque conductivity is given by

$$
\sigma_{x y}^{\tau}=\left(\sigma_{x y}^{\tau_{s}}+\sigma_{x y}^{\tau_{o}}\right)=\operatorname{Re}\left[i \partial i_{y}(\mathbf{q}) /\left.\partial q_{x}\right|_{q_{x} \rightarrow 0}\right],
$$

where $\chi_{y}(q)$ is defined as

$$
\begin{aligned}
\chi_{y}(\mathbf{q})= & \frac{i e \hbar}{V} \sum_{n \neq n^{\prime}} \sum_{\mathbf{k}}\left(f_{n \mathbf{k}}-f_{n^{\prime} \mathbf{k}+\mathbf{q}}\right) \\
& \times \frac{\left\langle n \mathbf{k}|\tau(\mathbf{k}, \mathbf{q})| n^{\prime} \mathbf{k}+\mathbf{q}\right\rangle\left\langle n^{\prime} \mathbf{k}+\mathbf{q}\left|v_{y}(\mathbf{k}, \mathbf{q})\right| n \mathbf{k}\right\rangle}{\left(E_{n \mathbf{k}}-E_{n^{\prime} \mathbf{k}+\mathbf{q}}\right)^{2}},
\end{aligned}
$$

where $\tau(\mathbf{k}, \mathbf{q})=\frac{1}{2}[\tau(\mathbf{k})+\tau(\mathbf{k}+\mathbf{q})], \mathbf{v}(\mathbf{k}, \mathbf{q})=\frac{1}{2}[\mathbf{v}(\mathbf{k})+\mathbf{v}(\mathbf{k}+\mathbf{q})]$ 
and $\tau=\frac{1}{i \hbar}\left[s_{z}+L_{z}, H_{0}\right]$ is the total torque. The equation (C1) suggests that we can choose $\mathbf{q}=\left(q_{x}, 0,0\right)$ for simplicity. By the choice of $\mathbf{q}=\left(q_{x}, 0,0\right)$, the $\chi_{y}(\mathbf{q})$ can be expanded in power of $q_{x}$, i.e., $\chi_{y}(\mathbf{q})=\chi_{y}^{(0)}+\chi_{y}^{(1)} q_{x}+\chi_{y}^{(2)} q_{x}^{2}+o\left(q_{x}^{3}\right)$, and the torque conductivity will be rewritten as $\sigma_{x y}^{\tau}=\operatorname{Re}\left[i \chi_{y}^{(1)}\right]$ $=-\operatorname{Im}\left[\chi_{y}^{(1)}\right]$ by using Eq. (C1).

To evaluate $\chi_{y}$ in Eq. (C2), we first expand the matrix elements $\left\langle n \mathbf{k}|\tau(\mathbf{k}, \mathbf{q})| n^{\prime} \mathbf{k}+\mathbf{q}\right\rangle\left\langle n^{\prime} \mathbf{k}+\mathbf{q}\left|v(\mathbf{k}, \mathbf{q})_{y}\right| n \mathbf{k}\right\rangle$ to first order of $q_{x}$

$$
\begin{aligned}
\langle n \mathbf{k}| & \tau(\mathbf{k}, \mathbf{q})\left|n^{\prime} \mathbf{k}+\mathbf{q}\right\rangle\left\langle n^{\prime} \mathbf{k}+\mathbf{q}\left|v(\mathbf{k}, \mathbf{q})_{y}\right| n \mathbf{k}\right\rangle= \\
& -\frac{n \beta \hbar}{m} k_{y} \frac{\partial \theta}{\partial k_{x}}\left(k_{x} \sin \theta+k_{y} \cos \theta\right) q_{x}-\frac{\lambda \beta}{\hbar}\left(k_{y}+k_{y} \cos (2 \theta)\right. \\
& +k_{x} \sin (2 \theta)+q_{x} \frac{\partial \theta}{\partial k_{x}} \\
& \left.\times\left[k_{x} \cos (2 \theta)-k_{y} \sin (2 \theta)\right]+\frac{q_{x}}{2} \sin (2 \theta)\right) \\
& -\frac{\beta^{2}}{\hbar}\left(k_{x}+k_{y} \sin (2 \theta)-k_{x} \cos (2 \theta)\right. \\
+ & q_{x} \frac{\partial \theta}{\partial k_{x}}\left[k_{y} \cos (2 \theta)+k_{x} \sin (2 \theta)\right] \\
+ & \left.\frac{q_{x}}{2}[1-\cos (2 \theta)]\right)+o\left(q_{x}^{2}\right) .
\end{aligned}
$$

We also need to expand the $\left(f_{n \mathbf{k}}-f_{n^{\prime} \mathbf{k}+\mathbf{q}}\right) /\left(E_{n \mathbf{k}}-E_{n^{\prime} \mathbf{k}+\mathbf{q}}\right)^{2}$ to first order of $q_{x}$. We have

$$
\begin{aligned}
\frac{f_{n \mathbf{k}}-f_{n^{\prime} \mathbf{k}+\mathbf{q}}}{\left(E_{n \mathbf{k}}-E_{n^{\prime} \mathbf{k}+\mathbf{q}}\right)^{2}}= & \frac{f_{n \mathbf{k}}-f_{n^{\prime} \mathbf{k}}}{\left(E_{n \mathbf{k}}-E_{n^{\prime} \mathbf{k}}\right)^{2}}+q_{x} \frac{\partial E_{n^{\prime} \mathbf{k}}}{\partial k_{x}} \\
& \times\left(2 \frac{f_{n \mathbf{k}}-f_{n^{\prime} \mathbf{k}}}{\left(E_{n \mathbf{k}}-E_{n^{\prime} \mathbf{k}}\right)^{3}}-\frac{\partial f_{n^{\prime} \mathbf{k}} / \partial E_{n^{\prime} \mathbf{k}}}{\left(E_{n \mathbf{k}}-E_{n^{\prime} \mathbf{k}}\right)^{2}}\right) \\
& +o\left(q_{x}^{2}\right) .
\end{aligned}
$$

Assuming the Fermi energy is lager than the spin-orbit splitting and using using $\left(k_{F}^{+}-k_{F}^{-}\right)=2 m \gamma(\phi) / \hbar^{2}$ and $\sigma_{x y}^{\tau}$ $=-\operatorname{Im}\left[\chi_{y}^{(1)}\right]$, we can write the torque conductivity as

$$
\sigma_{x y}^{\tau}=\frac{e \beta}{4 \pi^{2}} \int_{0}^{2 \pi} d \phi \frac{G(\lambda, \beta, \phi)}{\gamma(\phi)}-\frac{e \beta}{8 \pi^{2}} \int_{0}^{2 \pi} d \phi \frac{H(\lambda, \beta, \phi) \cos \phi}{[\gamma(\phi)]^{2}},
$$

where $G(\lambda, \beta, \phi)$ and $H(\lambda, \beta, \phi)$ are given by

$$
\begin{aligned}
G(\lambda, \beta, \phi) & =\frac{\left(\beta^{2}-\lambda^{2}\right) \sin ^{2} \phi[\lambda \sin (2 \phi)-\beta]}{[\gamma(\phi)]^{3}}, \\
H(\lambda, \beta, \phi)= & \lambda \sin \phi+\beta \cos \phi+\left(\lambda^{2}-\beta^{2}\right) \\
& \times \frac{(\lambda \sin \phi-\beta \cos \phi) \cos (2 \phi)}{[\gamma(\phi)]^{2}} \\
+ & \left(\lambda^{2}+\beta^{2}\right) \frac{(\lambda \cos \phi+\beta \sin \phi) \sin (2 \phi)}{[\gamma(\phi)]^{2}}
\end{aligned}
$$

$$
-2 \lambda \beta \frac{(\lambda \cos \phi+\beta \sin \phi)}{[\gamma(\phi)]^{2}} .
$$

Using $[\gamma(\phi)]^{2}=\left(\lambda^{2}+\beta^{2}\right)-2 \lambda \beta \sin (2 \phi)$, we find that $\frac{G}{\gamma(\phi)}$ $-\frac{H \cos \phi}{2 \gamma(\phi)^{2}}$ can be written as

$$
\left(\frac{G(\lambda, \beta, \phi)}{\gamma(\phi)}-\frac{1}{2} \frac{H(\lambda, \beta, \phi)}{\gamma(\phi)^{2}}\right)=\left(\beta^{2}-\lambda^{2}\right) \frac{2 \lambda \sin \phi \cos \phi-\beta}{\gamma(\phi)^{4}} .
$$

From the above formula, we can easily check that Eq. (C6) is zero when we set $\lambda^{2}=\beta^{2}$. From Eq. (C6), it follows that the torque conductivity vanishes in the pure Rashba system (i.e., $\beta=0)$ because the integral $\int d \phi \sin \phi \cos \phi$ is zero. Our next step is to work out the integrals by using the residue method. ${ }^{41}$ The crucial integrals are the following $(|\lambda|>|\beta|)$ :

$$
\begin{gathered}
\int_{0}^{2 \pi} d \phi \frac{1}{[\gamma(\phi)]^{4}}=\frac{2 \pi\left(\lambda^{2}+\beta^{2}\right)}{\left(\lambda^{2}-\beta^{2}\right)^{3}}, \\
\int_{0}^{2 \pi} d \phi \frac{\sin \phi \cos \phi}{[\gamma(\phi)]^{4}}=\frac{2 \pi \lambda \beta}{\left(\lambda^{2}-\beta^{2}\right)^{3}} .
\end{gathered}
$$

For $|\beta|>|\lambda|$, we can exchange the Rashba and Dresselhaus couplings $\lambda \leftrightarrow \beta$ in the integrals. Substituting Eq. (C6) and Eq. (C7) into Eq. (C5), we obtain the torque conductivity

$$
\sigma_{x y}^{\tau}=-\frac{e}{2 \pi} \frac{\beta^{2}}{\left|\lambda^{2}-\beta^{2}\right|} \text {. }
$$

Since the commutation relation $\left[s_{z}+L_{z}, \frac{p^{2}}{2 m}+H_{R}\right]=0$, the Rashba coupling disappears in numerator of $\sigma_{x y}^{\tau}$. For $\tau_{s}^{R}$ $\equiv \frac{1}{i \hbar}\left[s_{z}, H_{R}(\mathbf{k})\right]=\lambda\left(\sigma_{x} k_{x}+\sigma_{y} k_{y}\right)$, we can use the same steps as the calculation of $\sigma_{x y}^{\tau}$. The $H_{s}(\lambda, \beta, \phi)$ and $G_{s}(\lambda, \beta, \phi)$ can be obtained by changing the $\lambda$ and $\beta$ in $H(\lambda, \beta, \phi)$ and $G(\lambda, \beta, \phi)$. We have

$$
\begin{aligned}
\sigma_{x y}^{\tau_{s}^{R}}= & -\sigma_{x y}^{\tau_{o}^{R}}=-\frac{e \lambda}{8 \pi^{2}} \int_{0}^{2 \pi} d \phi \frac{G_{s}(\lambda, \beta, \phi)}{\gamma(\phi)} \\
& +\frac{e \lambda}{16 \pi^{2}} \int_{0}^{2 \pi} d \phi \frac{H_{s}(\lambda, \beta, \phi) \cos \phi}{[a(\phi)]^{2}} \\
= & -\frac{e \lambda}{8 \pi^{2}} \int_{0}^{2 \pi} d \phi \frac{G(\lambda \leftrightarrow \beta, \phi)}{\gamma(\phi)} \\
& +\frac{e \lambda}{16 \pi^{2}} \int_{0}^{2 \pi} d \phi \frac{H(\lambda \leftrightarrow \beta, \phi) \cos \phi}{[\gamma(\phi)]^{2}}=\frac{e}{4 \pi} \frac{\lambda^{2}}{\left|\lambda^{2}-\beta^{2}\right|} .
\end{aligned}
$$

Therefore, the spin torque Hall conductivity is

$$
\begin{aligned}
\sigma_{x y}^{\tau_{s}} & =\left(-\frac{e}{4 \pi} \frac{\beta^{2}}{\left|\lambda^{2}-\beta^{2}\right|}+\frac{e}{4 \pi} \frac{\lambda^{2}}{\left|\lambda^{2}-\beta^{2}\right|}\right)=\frac{e}{4 \pi\left|\lambda^{2}-\beta^{2}\right|} \\
& =\operatorname{sign}\left(\lambda^{2}-\beta^{2}\right) \frac{e}{4 \pi}
\end{aligned}
$$

and the OAM torque Hall conductivity is 


$$
\sigma_{x y}^{\tau_{o}}=\left(-\frac{e}{4 \pi} \frac{\beta^{2}}{\left|\lambda^{2}-\beta^{2}\right|}-\frac{e}{4 \pi} \frac{\lambda^{2}}{\left|\lambda^{2}-\beta^{2}\right|}\right)=-\frac{e}{4 \pi} \frac{\lambda^{2}+\beta^{2}}{\left|\lambda^{2}-\beta^{2}\right|} .
$$

For the pure Rashba system, we can take $\beta=0$ in the $\lambda^{2}$ $>\beta^{2}$ condition. For the pure Dresselhaus system, we can take $\lambda=0$ in the $\lambda^{2}<\beta^{2}$ condition.

\section{APPENDIX D: GAUGE INVARIANT POSITION OPERATOR}

In this appendix, we shall consider a method to obtain the gauge invariant position operator and resolve the problem that the expectation value of the OAM depends on the choice of the eigenstates in the absence of applied electric field. In crystalline environment, the position operator cannot be simply set to $\mathbf{x}=i \frac{\partial}{\partial \mathbf{k}} \cdot{ }^{42}$ We find that the OAM will depend on the choice of the eigenstates when we use the definition $\mathbf{x}=i \frac{\partial}{\partial \mathbf{k}}$. For example, the following states can also be chosen as the eigenstates:

$$
|n \mathbf{k} ; M\rangle=\frac{e^{i M(\mathbf{k})}}{\sqrt{2}}\left(\begin{array}{c}
1 \\
i n e^{i \theta(\mathbf{k})}
\end{array}\right),
$$

where $M(\mathbf{k})$ is the phase factor and $n= \pm 1$. It can be proved that the eigenstates of Eq. (D1) satisfy the relation $H_{0}$ $=\Sigma_{n} E_{n \mathbf{k}}|n \mathbf{k} ; M\rangle\langle n \mathbf{k} ; M|$ where the eigenenergy $E_{n \mathbf{k}}$ is defined in Eq. (7). Using the eigenstates of Eq. (D1), the matrix element $\left\langle n \mathbf{k} ; M\left|L_{z}\right| n \mathbf{k} ; M\right\rangle$ is zero if $M=-\theta / 2$ but nonzero if $M=-\theta$. Furthermore, if the eigenstates of Eq. (21) are chosen, the signs of $\left\langle+\widetilde{\mathbf{k}}\left|L_{z}\right|+\tilde{\mathbf{k}}\right\rangle$ and $\left\langle-\tilde{\mathbf{k}}\left|L_{z}\right|-\tilde{\mathbf{k}}\right\rangle$ will be different. Let us now consider the phase transformation of $|\overline{n \mathbf{k}}\rangle$ $=e^{i \Lambda_{n}(\mathbf{k})}|n \mathbf{k}\rangle$ where the phase $\Lambda_{n}(\mathbf{k})$ depends on band index $n$. We note that the eigenstates of Eq. (5) will be transformed to that of Eq. (21) by the appropriate choice of the phase $\Lambda_{n}$. Actually, we have $|+\tilde{\mathbf{k}}\rangle=(-i)|+\mathbf{k}\rangle$ and $|-\tilde{\mathbf{k}}\rangle=e^{i \theta}|-\mathbf{k}\rangle$. Let us also introduce the operator $\mathbf{X}^{\prime} \equiv \mathbf{x}+\mathbf{A}^{\prime}$ where $\mathbf{x}=i \frac{\partial}{\partial \mathbf{k}}$ and $\mathbf{A}^{\prime}$ is the vector potential. Using the above gauge transformation, ${ }^{26}$ we obtain

$$
\left\langle\overline{n \mathbf{k}}\left|\mathbf{X}^{\prime}\right| \overline{n \mathbf{k}}\right\rangle=\langle n \mathbf{k}|\mathbf{x}| n \mathbf{k}\rangle+\mathbf{A}^{\prime}-\frac{\partial \Lambda_{n}}{\partial \mathbf{k}} .
$$

Consider the specific transformation

$$
\mathbf{A}^{\prime}=\mathbf{A}+\frac{\partial \Lambda_{n}}{\partial \mathbf{k}}
$$

Equation (D2) becomes the gauge invariant form ${ }^{43}$

$$
\left\langle\overline{n \mathbf{k}}\left|\mathbf{X}^{\prime}\right| \overline{n \mathbf{k}}\right\rangle=\langle n \mathbf{k}|\mathbf{X}| n \mathbf{k}\rangle,
$$

where the operator $\mathbf{X}$ is defined as $\mathbf{X}=i \frac{\partial}{\partial \mathbf{k}}+\mathbf{A}$. We find that $\mathbf{A}^{\prime}$ satisfies the transformation (D3) if $\mathbf{A}^{\prime}$ is defined as

$$
\mathbf{A}_{n}^{\prime} \equiv\left\langle\overline{n \mathbf{k}}\left|(-i) \frac{\partial}{\partial \mathbf{k}}\right| \overline{n \mathbf{k}}\right\rangle
$$

which is the Berry vector potential when the eigenstates $|\overline{n \mathbf{k}}\rangle$ are used. The vector potential $\mathbf{A}$ in the eigenstates $|n \mathbf{k}\rangle$ can be defined as $\mathbf{A}_{n}=\left\langle n \mathbf{k}\left|(-i) \frac{\partial}{\partial \mathbf{k}}\right| n \mathbf{k}\right\rangle$ which generally depends on band index $n$. Therefore, the gauge invariant position operator $\mathbf{X}$ will depend on band index $n$,

$$
\mathbf{X}_{ \pm}=i \frac{\partial}{\partial \mathbf{k}}+\mathbf{A}_{ \pm}
$$

We find that the expectation value $\left\langle n \mathbf{k}\left|\mathbf{X}_{n}\right| n \mathbf{k}\right\rangle$ vanishes, $\left\langle n \mathbf{k}\left|\mathbf{X}_{n}\right| n \mathbf{k}\right\rangle=0$. It can also be shown that $\left\langle n^{\prime} \neq n \mathbf{k}\left|\mathbf{X}_{n}\right| n \mathbf{k}\right\rangle$ $=\left\langle n^{\prime} \neq n \mathbf{k}\left|i \frac{\partial}{\partial \mathbf{k}}\right| n \mathbf{k}\right\rangle$ by the use of $\left\langle n^{\prime} \mathbf{k} \mid n \mathbf{k}\right\rangle=\delta_{n^{\prime} n}$ where $\delta_{n^{\prime} n}$ is equal to unity for $n=n^{\prime}$ and zero for $n \neq n^{\prime}$. In terms of the gauge invariant position operator, the OAM operator $(\mathbf{x}$ $\times \mathbf{k})_{z}$ is replaced by $\left(\mathbf{X}_{n} \times \mathbf{k}\right)_{z}$. As a result, the expectation value of the OAM operator $\langle\mathbf{L}\rangle_{0}=\hbar\left\langle n \mathbf{k}\left|\mathbf{X}_{n}\right| n \mathbf{k}\right\rangle \times \mathbf{k}$ is zero for all the eigenstates in the absence of the applied electric field. Interestingly, note that the OAM operator does not always depend on band index $n$. For example, if the eigenstates of Eq. (5) are used, the Berry vector potential is independent of $n$, and so is the gauge invariant operator $\mathbf{X}_{n}$.

Let us now show that the calculated OAM Hall conductivities remain unchanged no matter whether the conventional $\mathbf{x}$ or gauge invariant $\mathbf{X}_{n}$ position operator is used. First of all, the gauge invariant velocity operator $\frac{1}{i \hbar}\left[\mathbf{X}_{ \pm}, H_{0}(\mathbf{k})\right]$ is the same as the original one $\frac{1}{i \hbar}\left[\mathbf{x}, H_{0}(\mathbf{k})\right]$ because the vector potential $\mathbf{A}_{ \pm}$commutes with $H_{0}(\mathbf{k})$, namely, $\left[\mathbf{A}_{ \pm}, H_{0}(\mathbf{k})\right]=0$. The gauge invariant OAM torque operator $\frac{1}{i \hbar}\left[\left(\mathbf{X}_{ \pm} \times \mathbf{k}\right)_{z}, H_{0}\right]$ is also the same as the conventional one $\frac{1}{i \hbar}\left[(\mathbf{x} \times \mathbf{k})_{z}, H_{0}\right]$ because $\left(\mathbf{A}_{ \pm} \times \mathbf{k}\right)_{z}$ commutes with $H_{0}(\mathbf{k})$. Nevertheless, the OAM current operator $\frac{1}{2}\left\{v_{x}, L_{z}\right\}$ has an extra term of $\left(\mathbf{A}_{ \pm} \times \mathbf{k}\right)_{z} v_{x}$ when the gauge invariant position operator $\mathbf{X}_{ \pm}$is used. For the eigenstates of Eq. (D1), we find that $\left\langle n \mathbf{k} ; M\left|v_{x}\right|-n \mathbf{k} ; M\right\rangle\left\langle-n \mathbf{k} ; M\left|v_{y}\right| n \mathbf{k} ; M\right\rangle=-\left[\left(\lambda^{2}\right.\right.$ $\left.\left.+\beta^{2}\right) \sin \theta \cos \theta+\lambda \beta\right] / \hbar^{2}$ and $\mathbf{A}_{ \pm}=\frac{1}{2}\left(2 \frac{\partial M}{\partial \mathbf{k}}+\frac{\partial \theta}{\partial \mathbf{k}}\right)$ are all purely real. ${ }^{44}$ On the other hand, Eq. (8) needs only the imaginary part of the matrix elements $\left(\mathbf{A}_{ \pm} \times \mathbf{k}\right)_{z}\left\langle n \mathbf{k} ; M\left|v_{x}\right|-n \mathbf{k} ; M\right\rangle$ $\times\left\langle-n \mathbf{k} ; M\left|v_{y}\right| n \mathbf{k} ; M\right\rangle$. This shows that the extra term $\left(\mathbf{A}_{ \pm}\right.$ $\times \mathbf{k})_{z} v_{x}$ does not contribute to the Kubo formula. In general, using $|\overline{n \mathbf{k}}\rangle=e^{i \Lambda_{n}}|n \mathbf{k}\rangle$, we have $\left\langle\overline{n \mathbf{k}}\left|v_{x}\right| \overline{n^{\prime} \mathbf{k}}\right\rangle\left\langle\overline{n^{\prime} \mathbf{k}}\left|v_{y}\right| \overline{n \mathbf{k}}\right\rangle$ $=\left\langle n \mathbf{k}\left|v_{x}\right| n^{\prime} \mathbf{k}\right\rangle\left\langle n^{\prime} \mathbf{k}\left|v_{y}\right| n \mathbf{k}\right\rangle$. With the eigenstates of Eq. (21), we obtain that $\left\langle\overline{n \mathbf{k} \mid}\left|v_{x}\right| \overline{-n \mathbf{k}\rangle}\right\rangle \overline{-n \mathbf{k} \mid} v_{y} \mid \overline{n \mathbf{k}\rangle}=-\left[\left(\lambda^{2}\right.\right.$ $\left.\left.+\beta^{2}\right) \sin \theta \cos \theta+\lambda \beta\right] / \hbar^{2}$ and $\mathbf{A}_{ \pm}=\mp \frac{1}{2} \frac{\partial \theta}{\partial \mathbf{k}}$, which are all purely real. Therefore, we conclude that the OAM Hall conductivities $\sigma_{x y}^{o_{0}}$ and $\sigma_{x y}^{\tau_{o}}$ remain the same even if we replace $\mathbf{x}$ with $\mathbf{X}_{+}$or $\mathbf{X}_{-}$. 
*Electronic address: gyguo@phys.ntu.edu.tw

${ }^{1}$ G. A. Prinz, Science 282, 1660 (1998); S. A. Wolf, D. D. Awschalom, R. A. Buhrman, J. M. Daughton, S. von Molnar, M. L. Roukes, A. Y. Chtchelkanova, and D. M. Treger, ibid. 294, 1488 (2001).

${ }^{2}$ I. Zutic, J. Fabian, and S. D. Sarma, Rev. Mod. Phys. 76, 323 (2004).

${ }^{3}$ S. Murakami, N. Nagaosa, and S.-C. Zhang, Science 301, 1348 (2003).

${ }^{4}$ J. Sinova, D. Culcer, Q. Niu, N. A. Sinitsyn, T. Jungwirth, and A. H. MacDonald, Phys. Rev. Lett. 92, 126603 (2004).

${ }^{5}$ M. I. D'yakonov and V. I. Perel, Sov. Phys. JETP 33, 1053 (1971); L. S. Levitov, Yu. V. Nazarov, and G. M. Eliashberg, ibid. 61, 133 (1985); J. E. Hirsch, Phys. Rev. Lett. 83, 1834 (1999); S. Zhang, ibid. 85, 393 (2000); L. I. Magarill, A. V. Chaplik, and M. V. Entin, Semiconductors 35, 1081 (2001).

${ }^{6}$ D. Culcer, J. Sinova, N. A. Sinitsyn, T. Jungwirth, A. H. MacDonald, and Q. Niu, Phys. Rev. Lett. 93, 046602 (2004).

${ }^{7}$ S. Zhang and Z. Yang, Phys. Rev. Lett. 94, 066602 (2005).

${ }^{8}$ G. Y. Guo, Y. Yao, and Q. Niu, Phys. Rev. Lett. 94, 226601 (2005).

${ }^{9}$ J.-I. Inoue, G. E. W. Bauer, and L. W. Molenkamp, Phys. Rev. B 70, 041303(R) (2004); S. Murakami, ibid. 69, 241202(R) (2004); E. G. Mishchenko, A. V. Shytov, and B. I. Halperin, Phys. Rev. Lett. 93, 226602 (2004); K. Nomura, Jairo Sinova, T. Jungwirth, Q. Niu, and A. H. MacDonald, Phys. Rev. B 71, 041304 (2005); R. Raimondi, and P. Schwab, ibid. 71, 033311 (2005); A. Khaetskii, Phys. Rev. Lett. 96, 056602 (2006).

${ }^{10}$ O. V. Dimitrova, Phys. Rev. B 71, 245327 (2005).

${ }^{11}$ O. Chalaev and D. Loss, Phys. Rev. B 71, 245318 (2005).

${ }^{12}$ J. Wunderlich, B. Kaestner, J. Sinova, and T. Jungwirth, Phys. Rev. Lett. 94, 047204 (2005).

${ }^{13}$ Y. K. Kato, R. C. Myers, A. C. Gossard, and D. D. Awschalom, Science 306, 1910 (2004).

${ }^{14}$ P.-Q. Jin, Y.-Q. Li, and F. C. Zhang, J. Phys. A 39, 7115 (2006).

${ }^{15}$ Q. F. Sun and X. C. Xie, Phys. Rev. B 72, 245305 (2005).

${ }^{16}$ P. Zhang, J. Shi, D. Xiao, and Q. Niu, cond-mat/0503505 (unpublished); J. Shi, P. Zhang, D. Xiao, and Q. Niu, Phys. Rev. Lett. 96, 076604 (2006).

${ }^{17}$ J. Wang, B. G. Wang, W. Ren, and H. Guo, cond-mat/0507159 (unpublished).

${ }^{18}$ Y. Wang, K. Xia, Z.-B. Su, and Z. Ma, Phys. Rev. Lett. 96, 066601 (2006).

${ }^{19}$ Y. A. Bychkov and E. I. Rashba, J. Phys. C 17, 6039 (1984).

${ }^{20}$ G. Dresselhaus, Phys. Rev. 100, 580 (1955).
${ }^{21}$ J. Nitta, T. Akazaki, H. Takayanagi, and T. Enoki, Phys. Rev. Lett. 78, 1335 (1997).

${ }^{22}$ M. Marder, Condensed Matter Physics (Wiley, New York, 2000).

${ }^{23}$ S.-Q. Shen, Phys. Rev. B 70, 081311(R) (2004).

${ }^{24}$ N. A. Sinitsyn, E. M. Hankiewicz, W. Teizer, and J. Sinova, Phys. Rev. B 70, 081312(R) (2004).

${ }^{25}$ M. V. Berry, Proc. R. Soc. London, Ser. A 392, 45 (1984).

${ }^{26}$ A. Bohm, A. Mostafazadeh, H. Koizumi, Q. Niu, and J. Zwanziger, The Geometric Phase in Quantum Systems (Springer, Berlin, 2003).

${ }^{27}$ J. Zak, Phys. Rev. Lett. 62, 2747 (1989).

${ }^{28}$ M. C. Chang and Q. Niu, Phys. Rev. Lett. 75, 1348 (1995); M. C. Chang and Q. Niu, Phys. Rev. B 53, 7010 (1996).

${ }^{29}$ S. Murakami, Naoto Nagaosa, and S. C. Zhang, Phys. Rev. B 69, 235206 (2004).

${ }^{30} \mathrm{We}$ evaluate $\sigma_{y x}^{s_{0}}$ and $\sigma_{y x}^{o_{0}}$ directly, and find the relations of $\sigma_{x y}^{s_{0}}$ $=-\sigma_{y x}^{s_{0}}$ and $\sigma_{x y}^{o_{0}}=-\sigma_{y x}^{o_{0}}$.

${ }^{31}$ D. Schmeltzer, cond-mat/0509607 (unpublished).

${ }^{32}$ M. C. Chang, Phys. Rev. B 71, 085315 (2005).

${ }^{33} \mathrm{~J}$. Hu, cond-mat/0503149 (unpublished).

${ }^{34}$ J. Li, L. Hu, and S.-Q. Shen, Phys. Rev. B 71, 241305(R) (2005).

${ }^{35}$ B. K. Nikolic, L. P. Zarbo, and S. Welack, Phys. Rev. B 72, 075335 (2005).

${ }^{36}$ J. J. Sakurai, Advanced Quantum Mechanics (Addison Wesley, New York, 1997).

${ }^{37}$ F. Meier and D. Loss, Phys. Rev. Lett. 90, 167204 (2004).

${ }^{38}$ N. Sugimoto, S. Onoda, S. Murakami, and N. Nagaosa, Phys. Rev. B 73, 113305 (2006).

${ }^{39}$ J. Schliemann, J. C. Egues, and D. Loss, Phys. Rev. Lett. 90, 146801 (2003)

${ }^{40}$ S. D. Ganichev, V. V. Bel'kov, L. E. Golub, E. L. Ivchenko, Petra Schneider, S. Giglberger, J. Eroms, J. De Boeck, G. Borghs, W. Wegscheider, D. Weiss, and W. Prettl, Phys. Rev. Lett. 92, 256601 (2004).

${ }^{41}$ G. Arfken and H. J. Weber, Mathematical Methods for Physicists (Academic, New York, 1995).

${ }^{42}$ E. I. Blount, in Formalisms of Band Theory in Solid State Physics, edited by F. Seitz and D. Turnbull (Academic, New York, 1962), Vol. 13, p. 305.

${ }^{43}$ J. J. Sakurai, Modern Quantum Mechanics (Addison Wesley, New York, 1994).

${ }^{44}$ In general, it can be proved that $\left\langle n \mathbf{k}\left|\frac{\partial}{\partial \mathbf{k}}\right| n \mathbf{k}\right\rangle$ is purely imaginary by means of $\langle n \mathbf{k} \mid n \mathbf{k}\rangle=1$ (see Ref. 26). In our definition of $\mathbf{A}_{n}$ $=\left\langle n \mathbf{k}\left|(-i) \frac{\partial}{\partial \mathbf{k}}\right| n \mathbf{k}\right\rangle$, however, $\mathbf{A}_{n}$ is purely real. 